\title{
How soft materials control harder ones: routes to bioorganization
}

\author{
Marshall Stoneham \\ London Centre for Nanotechnology and Department of Physics and Astronomy, \\ University College London, WC1E 6BT, London, UK
}

Received 9 January 2007, in final form 22 March 2007

Published 8 June 2007

Online at stacks.iop.org/RoPP/70/1055

\begin{abstract}
Ordered structures are remarkably common, even without direct human guidance or direction. The ordering can be at the atomic scale or on the macroscopic scale or at the mesoscale. The term 'self-organization' is often used, but this description is facile, giving no hint as to the range or variety of mechanisms. Ordering can occur in circumstances commonly associated with disorder, as in the irradiation of metals to high doses; it can also occur when soft, flexible materials organize structures of harder, rigid structures. My review attempts to analyse some of these widely varying behaviours, both to seek evidence of common underlying principles and to assess how organization might be controlled, and with what level of accuracy.
\end{abstract}




\section{Contents}

1. Making sense of organization 1057

2. Organization at the molecular or the crystal structural level 1059

2.1. Nucleation 1059

2.2. Multilayer growth 1060

2.3. Graphoepitaxy and structured substrates 1062

2.4. Vernier and moiré structures 1063

2.5. Biomineral selectivity on soft organic substrates 1064

3. Organization at the mesoscopic scale 1065

3.1. Qualitative aspects of organized structures 1065

3.2. Bringing order to organization 1068

3.3. Organization mechanisms 1069

3.4. Characteristic features of periodic structures 1071

3.5. Key characteristics of periodic organized structures 1082

4. Other issues, including robustness, self-organization and chirality 1084

4.1. Volume constraints and limits to growth 1084

4.2. Selectivity in the presence of fluctuations 1087

4.3. Robustness 1087

4.4. Chirality 1088

5. Conclusions: bringing organization to self-organization 1089

Acknowledgments $\quad 1091$

References 1091 


\section{Making sense of organization}

Nature manages to uses soft, flexible materials in ways that, at least at the macroscopic level, we associate with stiff, rigid structures. Examples include soft templates for the growth of an inorganic crystal with specific facets and orientations or the growth of small nanocrystals of controlled size. Then there is the growth of structures such as shells or bones whose complex hierarchical structure is important functionally (Stoneham and Harding 2003 and references therein). A distinct but important class concerns the various nanomotors that efficiently convert modest amounts of energy into motion (Howard 2001, Jones 2004).

In my analysis, I shall include examples of both hard and soft systems and from both living systems and those that are definitely not living. The aim is to understand phenomena associated with those many 'soft' biological materials that lack stiffness and rigidity. Softness in this sense can be fully consistent with incompressibility. Water is relatively incompressible, but not at all stiff, and water has a range of novel physical properties that appear to make it uniquely necessary for life (Finney et al 2004). Only a few of the mechanisms discussed seem to exploit the special capabilities of DNA directly. For this reason, it seemed helpful as context to include an analysis of the rather many mechanisms that give ordered structures, often periodic, in inorganic systems.

Organization has its own variants, although these are often linked. In understanding the principles, it can be useful to separate three types of organization. The first is organization at the atomic scale, when a particular crystal structure is selected. This selection step may involve a choice of chirality, a choice of surface plane (or planes if the main surface is faceted) or a choice between structures (e.g. wurtzite versus zincblende) or polytypes. Such selectivity has been exploited in the purification of pharmaceuticals. The mode of growth may involve island formation, either initially or after a small number of monolayers have been established. Often, the notion of a template is introduced. The selectivity might be at the nucleation stage or the growth stage. A subtle example is discussed by Mackay (1986), who describes a number of generalizations of crystallography that, in some cases, relate to organization in living systems. One such example stems from the work by Cairns-Smith (1982) indicating that kaolin plates can retain information in their $\mathrm{Al}, \mathrm{Si}$ atom pattern that can be replicated in the growth of new crystallites from the initial seeds. Cairns-Smith suggested this (inorganic) mechanism played a key role in the development of the (organic) DNA/protein-based replication that underpins evolution. He notes that the aluminosilicates provide an example of a self-replicating system, as suggested by von Neumann.

The second type of organization is mesoscopic in scale and leads to ordering. The most evident forms of ordering lead to structures periodic at the nanometre or the micrometre scale but there are other systematic forms, such as the 'Fibonacci' features evident in spiral structures, and even human fingerprints show systematic - if very individual-mesostructures. I shall start from the view that, when there are highly regular (e.g. periodic) structures and similar but less-well organized mesostructures on the same scale, then they have their origins in similar mechanisms.

The third type of organization leads to specific shapes (usually external shape), primarily at the larger mesoscale or the macroscale. The structure may be relatively soft, such as in some cell structures, where topology is crucial, or it may be stiff, as in bone or shell. What is observed often suggests the concept of a volume constraint, although it is not always clear how a soft solid can offer a strong volume or shape constraint to a hard material. Moreover, even hard structural materials, such as concrete, have problems in constraining the growth of apparently soft plants. So the question remains: how can a soft-shaped solid constrain growth of a hard, rigid, solid to a desired shape? The 
underlying issue is how growth can be restricted in a controlled way and is addressed in section 3.

Symmetry plays a crucial role at the atomic scale, and both symmetry and symmetry breaking can be critical at the mesoscale (see, e.g. Weyl (1952), Thom (1983)). At the macroscopic scale, the roles of history, environment and evolutionary advantage through selection can control symmetry. For example, a recent newspaper report (Metro 27 March 2006) suggested that snails choose a left-handed spiral to make it hard for right-handed crabs to kill them. Strictly, this only points to probable correlations between the handedness of snails and of crabs, and one might ask about those other foods to which the crabs had access. As regards symmetry breaking, the catastrophe theory of Thom develops important ideas as to the classification of models of morphogenesis. Complex patterns can be generated reproducibly (Koch and Meinhardt 1994, Meinhardt 1992), including periodic patterns of units that have a complex and polar substructure, such as photoreceptor cells in the Drosophila eye. The patterns that form occur both in animals, e.g. the fly's eye, and in plants, e.g. how leaves and auxiliary buds are placed in a growing shoot.

Spatial organization has its own timescales. Some are related to characteristic length scales, e.g. through a diffusion constant. Processes leading to organization can be incoherent, e.g. as a system evolves to a minimum free energy. Synchronicity is seen in many natural systems, leading to systematic phase relations in situations as varied as light emission by fireflies, chemical oscillations, laser systems and synchronised chaos. Time concordance does not seem an issue in the cases discussed in this survey, but is described elsewhere (Strogatz 2003, Winfree 1980).

The variety of the possible underlying mechanisms of bioorganization is striking. Each field has its own fashions. Even though 'self-assembly' or 'self-organization' are often credible descriptions, the mechanisms that underly them are very diverse. Some key ideas emphasize a final structure, others the mechanism by which that structure is achieved. There are more complex issues of chirality, selectivity and how robustness is maintained when there are fluctuations, as there will always be at the nanoscale. Sometimes the ordering arises through a scaffold or template that may or may not survive the creation of the final structure. Certain mechanisms imply a scaling law or relationships between different characteristic lengths. However, this is by no means universal, and any claims that a system appears to be fractal imply there is no scale length.

Much of this survey intentionally concerns organization, rather than bioorganization. It is widely appreciated that advanced life forms achieve marvels of organization by means of the subtle and ingenious use of DNA, RNA and proteins. This organization can be at the molecular scale, as in highly selective receptors, or at the mesoscale, as in cell structures, or at the macroscopic scale, as in skeletal or circulatory systems. Gould (1991) has observed that D'Arcy Thompson's seminal approach attempts to see how far physical arguments can take us. There is no need to take one or other of the extreme views that propose either that the constraints imposed by simple physical arguments dominate growth and organization or alternatively that genetic coding can 'decide' everything. But the mechanisms by which living organisms achieve their complex structures have to adopt the same physical and chemical principles that are available in simple physical or chemical systems. The so-called programmed organization (as in man-made structures, at one extreme) has to follow natural physical laws. As this survey should make clear, there is abundant evidence for organization at the mesoscale in, for example, highly-irradiated solid metallic elements. How much of bioorganization requires the special properties of DNA, RNA and proteins? Do these very special molecules of life control organization or do they fine tune the organization that arises from simple physical mechanisms? My immediate aim is to survey ordering behaviours, especially in those where neither DNA 
nor proteins are involved, identifying examples of ordering that arise in a natural-if often non-intuitive-manners. Nature is very effective in realizing complex systems in controlled and reproducible manners. Understanding the underlying mechanisms for simpler physical systems should aid the modelling of ordering behaviour and should provide a basis for assessing the degree of control that may prove possible in man-made systems.

\section{Organization at the molecular or the crystal structural level}

\subsection{Nucleation}

Organization often nucleates locally and then spreads, so aspects of nucleation will appear at many points in this paper. Even when primary order arises in other ways (e.g. when short-range order precedes long-range order), nucleation may be involved in some other way, perhaps by controlling a phase. This section addresses some of the basic ideas.

Crystal growth is perhaps the classic example of nucleation theory (e.g. Tiller 1991). In its simplest and commonest form, nucleation theory identifies two main terms in the energy of a nucleus with a characteristic length $L$. One energy is a volume term scaling as $L^{3}$, the other an interface term scaling as $L^{2}$. For $L$ greater than a critical scale length $L_{\mathrm{c}}$, it is energetically favourable for the nucleus to grow. Simple generalizations take account of relative surface energies (the surface stress seems far less important) for different facets, but generally assert a drive to a minimum free energy. Other generalizations recognise the existence of a substrate and of features on that substrate (which may constitute a template) and the additional energies involved (strain and electrostatic being common contributions). The picture just given is often generalized, e.g. to account for the dependences of surface energies on the underlying crystal structure and how different crystal forms and morphologies (e.g. Bennema (1991), citing Burton et al (1951), Cahn et al (1991) citing Frank (1958, 1972)) arise. Even these modest extensions can be far too simple, of course. Frank has shown that kinetic effects can affect nucleation rates dramatically, e.g. through the role of screw dislocations. Or the system may be manoeuvred towards a quite different structure by apparently minor factors, e.g. in high dose radiation damage of metals, free energy arguments point to dislocation loops, yet He from $\alpha$ particles can stabilize the alternative void structure at a particular critical size.

Even in the simplest nucleation picture, there is an energy barrier to nucleation: at critical length scale $L_{\mathrm{c}}$ the sum of the interfacial and the bulk energy terms is a maximum. Nucleation is energy intensive, and accelerating this step can have a dramatic effect on crystallization rates. This has led to laser-stimulated nucleation and to the use of an intense acoustic field in a closed vessel to stimulate nucleation (sonocrystallization). Such approaches offer powerful ways to ensure that a single pure crystal form is created, often with advantageous size distributions. They are especially important for drug manufacturers seeking a pure product (e.g. Banga et al 2004, Dennehy 2003). It is conceivable that some of the commercial 'water softener' devices use turbulence to ensure a specific crystal morphology.

The nature of the fluctuations can be crucial. Indeed, protein crystal nucleation may be substantially enhanced by critical density fluctuations (den Wolde and Frenkel 1997). The 'thermodynamics versus dynamics' argument is encountered in activated processes such as nucleation and diffusion. There will be a first passage theory (e.g. Rice-Slater dynamical theory) and a thermodynamic theory based on an ensemble for which one compares populations of different regions of phase space (e.g. Vineyard theory or reaction rate theory; see, e.g. Itoh and Stoneham (2001), section 3.4.4) or alternatively the probability that fluctuations will permit systems from one defined region of phase space to reach another defined region. These are not equivalent. Nucleation theory always seems to follow the thermodynamic arguments. But 
the usual assumptions about length and time scales may be simplistic for soft systems with large thermal fluctuations; one should recall that at room temperature, a 100-atom system will have root mean square volume fluctuations of order $1 \%$. Thermal fluctuations are sufficiently significant that, in living things, nature does not seem to attempt to achieve perfect fidelity using repair enzymes (Radman 2001), a situation far removed from average solid-state modelling. Nor does nature concentrate on high symmetry nuclei with only a single scale length as control parameter. This natural flexibility is found even in inorganic systems. For example, vacancies nucleate to form 3D voids in metals under irradiation, even though this $3 \mathrm{D}$ structure is more costly in energy than the 2D planar dislocation loops. A crucial factor appears to be the capture of $\mathrm{He}$ ( $\alpha$ particles) at an early stage (possibly when a mere four vacancies have aggregated), the gas atom driving the $3 \mathrm{D}$ structure to beyond a critical size. In other words, relatively modest factors can bias evolution towards an unexpected structure that is not that of lowest free energy. Kinetic trapping is also apparent in the growth of $\mathrm{ZnO}$ in the polar $\left(\begin{array}{llll}0 & 0 & 0 & 1\end{array}\right)$ direction, despite the high energy of this surface. In this case, kinetic trapping occurs because the initial structure is graphitic where the $(0001)$ face is of lowest energy; when the growing system transforms to the zincblende structure, there is no reasonable pathway to a growth direction with a low energy surface (Freeman et al 2006).

\subsection{Multilayer growth}

Commensurate and weakly incommensurate structures. The key factors in multilayer growth are associated with misfit: strain energy and ideas of interfacial dislocations. The seminal work of Frank and van der Merwe (1949a, 1949b) is reviewed in van der Merwe (1991), where he also discusses the conditions for layer-by-layer growth, island growth (Volmer-Weber) and layer-by-layer growth transforming to islands (Stranski-Krastanov). The initial analysis was stimulated by Royer (1928) noting epitaxy only when the misfit is less than $15 \%$ and by Finch and Quarrell (1934) observing that $\mathrm{ZnO}$ grown on $\mathrm{Zn}$ strains homogeneously to match the Zn substrate.

Frank and van der Merwe's work also proved seminal as the technological advantages of strained layer semiconductor systems became evident. Stoneham and Jain (1995) gave details of pioneering papers, with comments on dislocation nucleation. The original ideas of Shockley's 1951 patent were realized in works by Kasper et al (1975) and Kasper and Herzby (1977). The early equilibrium theories (energy minimization with respect to strain; Ball and van der Merwe (1983) gave fuller references) were reformulated in terms of force balance on a dislocation (Matthews and Blakeslee 1977). Jain et al (1992) showed that it was crucial to include the interactions between the dislocations. For self-organized quantum dots (typically ten thousand atoms, III-V or group IV semiconductors) the theory is now both sophisticated and successful through close links with experiment. There continue to be controversial aspects, such as the extent to which line tensions are important, as well as surface energies. Overgrowths of silicon on self-ordered islands of $\mathrm{Ge}$ on $\mathrm{Si}\left(\begin{array}{l}0 \\ 0\end{array} 1\right)$ affect the lateral ordering through several mechanisms (Capellini et al 2006), causing a shape transformation from domes to truncated pyramids of larger base. One important factor is the gradient in chemical potential across the island, which drives a selective flow of both $\mathrm{Ge}$ and $\mathrm{Si}$ atoms at the surface and, in turn, causes the dots to move so as to favour lateral order.

Templating. The idea of a template is not restricted to crystal-on-crystal situations. The widely used Frenkel Kontorova model (Frenkel and Kontorova 1938) describes harmonically coupled particles and how they can minimize energy on a substrate with a sinusoidal potential. In one dimension, the predicted critical energies are asymmetric with respect to the sign of 
the mismatch (Milchev and Markov 1984). The Frenkel-Kontorova model shows a wealth of behaviour and has been used extensively for monolayers of adatoms on incommensurate surfaces. Even monolayers on surfaces offer a rich variety of organized behaviours (Rosei 2004), reflecting different interactions with the substrate and between adatoms or groups of adatoms. The substrate interaction might be of the Frenkel-Kontorova form (Thayer et al 2002, Thürmer et al 2004; see also section 2.2) or more complex. The interactions between adatoms may involve simple short-range forces, or may be long-ranged (Zeppenfeld 2004, Zeppenfeld et al 1994), including elastic interactions (e.g., Stoneham 1977, Rickman and Srolovitz 1993, Pohl et al 1999, Longo et al 2006) that may involve surface steps (e.g., Marchenko and Parshin 1981). The surface species may show self-organization (e.g., Croset et al 2002), and there can be domain structures (van Gastel et al 2003). Such structures may offer scope for novel statistical mechanics, e.g., the commensurate/incommensurate transitions of films physisorbed on anisotropic substrates (Haldane and Villain 1981). However, some of the most significant cases involve relatively large molecules (e.g. polymer strands or rod-like micelles) on surfaces that impose some structure. De Gennes (1986), for example, has looked at how flexible polymer coils can recognize patterns on a surface. In such cases, there is the possibility that the polymer may form knots or show other topological features. This is especially the case for long molecules (Frisch and Wasserman 1961, Delbruck 1962, Sumners and Whittington 1988 ) and is the origin of the so-called 'Murphy's Law of Rope' (Matthews 1995). Rod-like surfactant micelles are observed to form ordered arrays on crystalline substrates, even though the micelles are much larger than the template unit cell. Molecular-scale interactions prove far too small to explain the alignment (Saville et al 2006), which arises through the van der Waals torque and Brownian motion as a dynamic process involving many molecules. At a still larger scale, the dynamics of giant $(10-100 \mu \mathrm{m})$ vesicles decorated with specific stickers adhering to bioactive substrates correlates strongly with the tension in the bounding lipid layer (Cuvelier and Nassoy 2004, Puech et al 2006). Spreading and diffusion are key processes in vesicle adhesion. There is a specificity, hence a time dependence, depending strongly on the chemical preparation of the substrate. These adhesion events affect cancer propagation and cell behaviour such as healing processes.

It is prudent to distinguish between templates, which provide a passive, usually geometric, guide to the selection of one structure over another, and the reactions that occur selectively at a receptor. When a specific molecule interacts with its receptor, it actuates some process. This actuation step is often hidden by a belief that molecular shape is all that matters. Certainly the molecular shape must fit the receptor well enough, in some sense, but the fit alone does not guarantee actuation. In the well-known 'lock and key' metaphor, one must have some action corresponding to turning the key. This can be a very helpful analogue for large molecules, where there may be regions that either encourage or avoid local contacts. For small molecules, say 50 atoms or less (and all olfactants and many neurotransmitters are in this class) may be a very incomplete description. Concentrating on the accuracy of the geometric fit at a receptor may be misleading since, for example, utterly different scents can be discerned even when the molecules have the same shape to high accuracy. For receptors, a better metaphor may be the 'swipe card': the shape has to be accurate enough, but the information that actuates the system is something else, typically magnetic for a real human-scale swipe card. For a receptor/molecule system, again the shape must be good enough, but the information that decides whether the molecule is right or wrong to actuate the system is carried in another way (Stoneham and Gavartin 2007, Brookes et al 2007). Turin's (1996) ideas of the role of inelastic electron tunnelling in olfaction would be an example of a swipe card mechanism, when an olfactant molecule's vibrational frequencies determine acceptability. In other systems, proton transfer may actuate the receptor (e.g. Kirby 1993, Wallace et al 1993). 
Families of structures. There are many families of closely related structures, with small energy differences between structures, e.g. the polytypes of SiC. The selection of one structure rather than another can be subtle. Systems such as some of the various tetrahedral (often aluminosilicate) structures (Wells 1986) and zeolites can involve a template of some sort. For some such systems, the natural structural unit is a polygon or a substantial unit typically of tens of atoms. One set of rules describes the structure of the polygon, and another set determines how the polygons are assembled or linked. This level corresponds to the classification of polytypism in spinelloid structures (Price 1983) and provides a basis for their simulation (Price et al 1985). It allows the classification of minerals by the 'polymerization' of coordination polyhedra (e.g. Hawthorne 1985), the classification of zeolites by their cuboctahedral links via sixmembered rings (e.g. zeolite A) or via four-membered rings (e.g. zeolite X, Y). The ideas of Cairns-Smith, noted above, propose that clay (an aluminosilicate that embodies substantial structural information and that can be replicated to some extent) provided crucial templates in the origin of life. He argues that clay could fulfil some of the functions of genes before the advent of DNA and RNA. Such ideas involve structural information being transferred across a hard/soft interface, allowing replication in a way that is somewhat analogous to the way that complex man-made structures exploit scaffolding that can be taken away later. In an analogous way, there might be 'low-tech' genetic materials enabling relatively sophisticated replication. In particular, the Earth makes clay all the time: perhaps aluminosilicates could be the catalysts for subsequent high-tech organic systems (and here we have the reverse of biomineralization)? Whether or not his ideas describe correctly how life did evolve, they do illustrate how simple ideas of inorganic ordering might have profound biological consequences.

\subsection{Graphoepitaxy and structured substrates}

In graphoepitaxy, a film grows on a structured substrate. A familiar example occurs when oriented ice crystals grow on small scratches on a car windscreen. Normally, graphoepitaxy relies on topographic features but, of course, the features could be associated with electric charge, with adhesiveness or with some other contrast across the interface.

For one surprising case, straightforward Monte-Carlo methods can give useful information. There is the remarkable observation (Cahn 1995, Shirikoff and Erb 1994) of an epitaxial relation between the substrate crystal $\mathrm{C}^{\prime}$ and the deposited crystal $\mathrm{C}^{\prime \prime}$ when there is an intermediate amorphous layer $\mathrm{A}$ (i.e., a $\mathrm{C}^{\prime \prime} / \mathrm{A} / \mathrm{C}^{\prime}$ structure). The key assumption (Stoneham and Torres, unpublished work 1995, Stoneham 2003) is that the substrate $\mathrm{C}^{\prime}$ has crystallographically determined surface features, like steps. The surface topography of the amorphous layer A maintains some memory of these steps, which guide the crystallographic orientation of $\mathrm{C}^{\prime \prime}$. Monte-Carlo calculations support this idea for nanoscale amorphous layers. What this suggests is that surface topography at above the atomic scale may be important (this is almost a mix of atomic scale templating and mesoscale space constraints). Analogous surface roughness factors lead to alignment of liquid crystals on polymer substrates that have been exposed to UV or are mechanically buffed (Kumar et al 2005). Kumar et al (2005) show that the anisotropy in the surface roughness of the substrate completely determines the direction of the LC alignment. This may be contrasted with systems in which molecules simply grow vertically from a surface, albeit with orientational order, e.g. cytosine and cytidine 5 -monophosphate at the $\mathrm{Au}\left(\begin{array}{lll}1 & 1 & 0\end{array}\right) 1 \times 2$ electrolyte interface (Weightman et al 2006).

Mother-of-pearl has an ordered, layered arrangement of $\mathrm{CaCO}_{3}$ platelets (aragonite structure). These abalone nacre structures have exceptional mechanical strength. They are grown by what seems an epitaxial process in a soft organic matrix of chitin and proteins. However, there is a continuous $3 \mathrm{~nm} \times 5 \mathrm{~nm}$ amorphous $\mathrm{CaCO}_{3}$ layer continuous coating 
(Nassif et al 2005, Burgess 2005). Mann et al (1995) (see also Bradley (1995)) postulated an organic (protein?) inter-crystal material. They note that, normally, $\mathrm{CaCO}_{3}$ forms long needles, but that additives have profound effects. Thus, adding a little Li leads to the hexagonal crystals such as those found in shellfish; adding organics such as aspartic acid gives strange shapes with spiralling protuberances. The idea proposed by Mann, and widely accepted, is that the polymer and the carbonate form a gel that coats the growing crystal, so the intercrystalline regions are substantially organic. The soft gel provides a means to grow shaped structures inside a template. There is a further idea needed here, since the amorphous-coated crystal 'bricks' actually form quite a well-ordered structure at the mesoscale (rather like a brick wall, in fact). So what causes this meso organization? The scale seems somewhat too large for simple Coulomb interactions, but that needs checking. Further studies of the templating of calcite and amorphous calcium carbonate films are given by Cavalli et al (2006a, 2006b) and Popescu et al (2006).

Phase separation of block copolymers provides one systematic way of generating patterned substrates with nanoscale features (e.g. Krishnamoorthy et al 2006). In block copolymers, two or more polymer chains are chemically bound to each other. Different polymers are reluctant to mix for entropic reasons, tending to form separate phases, especially for higher molecular weight cases. For block copolymers, phase separation must be intermolecular, with separations comparable to the chain size, a constraint that leads to separation into nearly periodic microphases. The domains are typically tens or hundreds of $\mathrm{nm}$ across. Many examples have been demonstrated in a wide range of polymer systems.

It is tempting to think of structure in a substrate as being a perturbation on a twodimensional space and to use a description based on roughness. Page and Sear (2006) (also Frenkel (2006)) have suggested that pores-which undoubtedly introduce the third dimension - provide a mechanism for protein crystallization. In essence, the pore walls attract molecules weakly, and crystal growth is initiated at corners. In due course, another nucleus can form where the structures in the pore meet the surface, allowing 'breakout' from the pore. The initial nucleation and subsequent breakout will have different timescales.

\subsection{Vernier and moiré structures}

Vernier patterns arise when two periodic structures with slightly different scale lengths are superimposed. In one dimension, suppose one structure has scale length $L$ and the other $L+\delta L$. There is then a pattern visible with period $L / \delta L$. Whether or not this pattern will be easy to see will depend on how near to integral is $L / \delta L$ and the extent to which the system can relax. The word 'moiré' is not named after a person, but comes from moire antique (Frank 1983 ) in which a watered fabric (usually silk) is produced by passing two sheets of cloth together between smooth hot rollers. Moiré fringes and similar patterns in science usually relate to larger scale features seen when two identical patterns (often periodic, though others are known) are superimposed with a slight displacement and relative rotation (e.g., Geiger 1986).

Thin layers of one material growing on a substrate that has its own structure provide one situation in which Vernier or moiré patterns can be seen. The substrate structure may be its atomic scale crystal structure, and one has the standard questions of epitaxy and of different growth modes (layer by layer, layer then islands, islands); see section 2.2. When the substrate has a superimposed, larger scale, possibly artificial structure, one has graphoepitaxy (see section 2.3).

The interference of waves offers another route to structures that look somewhat similar to Vernier or moiré patterns. Common cases involve waves on a liquid that impacts on a solid, 
e.g. in sand or pebble beds at sea fronts. Laser annealing sometimes shows related phenomena through waves stimulated in the transient fluid surface region. In the case of laser annealing, the situation is dynamic: waves traverse the fluid layer as it is solidifying from the substrate side.

\subsection{Biomineral selectivity on soft organic substrates}

Living organisms are able to control sizes, shapes and crystallographic orientation of growing crystals to form minerals with unusual morphologies and functional properties (Mann 2001, Weiner and Addadi 1997, Addadi and Weiner 1997). Brittlestars, marine relatives of starfish, contain calcite crystals that act both as armour and as optical receptors for the one compound eye. Not only do the lenses focus light about $5 \mu \mathrm{m}$ below the surface but they are shaped to compensate for birefringence and spherical aberration (Aizenberg et al 2001). Similar phenomena are noted for trilobites (Forty 2001, Clarkson and Levi-Setti 1975).

Laboratory efforts mimic such biological controls have simpler aims and commonly exploit Langmuir monolayers, in which long-chain carboxylic acids (Mann et al 1991, Heywood and Mann 1994) form ordered arrays on the surface of water or self-assembled monolayers grown on substrates, typically gold films. The substrate can control the surface of calcium carbonate which nucleates. The different head groups of self-assembled monolayers tend to nucleate different crystal faces (Küther et al 1998, Aizenberg et al 1999a, 1999b). Calcite crystals grown from an aqueous solution on Langmuir monolayers nucleate on the (10.0) face, whereas those on the surface of pure water nucleate on the (10.4) face. Even different metal substrates can make a difference. Polymer templates such as dendrimers of poly-amidoamine induce the growth of spherical or disc-like crystals (Naka and Chuo 2001). Poly-pentacosadiynoic acid (PDA) promotes nucleation of the (01.2) face of calcite (Berman and Charych 1999). The flexibility of these monolayers is observed to be important in controlling growth for bis-urea layers with different side groups to control their stiffness. Increased flexibility correlates with increased orientation control (Cavalli et al 2006a, 2006b, Popescu et al 2006).

Flexible organic layers and scaffolds clearly control the growth of minerals in biology (Harding and Duffy 2006). Growth usually occurs at chemically active surfaces in contact with water. The processes controlling the details of crystal growth involve competition with pre-existing water molecules, ionization of the monolayer and the arrangement and the density of surface functional groups. It is only during crystal growth that there is detailed matching between the crystal and the organic film. This mixture of phenomena on several length scales can be modelled and certainly needs computer simulations based on large scale molecular dynamics. But an other theory is needed as a framework, including classical nucleation theory and the Wulff-Kaishew theorem of equilibrium crystal shapes. Duffy and Harding (2002, 2004a, 2004b, 2005a, 2005b) predict the morphologies of calcite crystals grown on stearic (octadecanoic) acid monolayers and find good agreement with experiment. They find it essential to go beyond models based on the simplest template ideas (figure 1). The organic substrate does not merely provide a pattern that the growing crystal must fit. The organic monolayers are not rigid structures in a vacuum, and factors such as the state of ionization of the monolayer and the density of surface carbonate ions are much more important than detailed stereo-chemical matching in controlling crystal morphology. That simple templating ideas fail is demonstrated when calcite crystals are nucleated from the supersaturated solution on top of alkylthiols with carboxylic acid head groups that have been self-assembled on gold substrates. The calcite crystals exhibit a strong tendency to nucleate on the $\left(\begin{array}{llll}0 & 1 & 1 & 2\end{array}\right)$ calcite face (Travaille et al 2002, 2003), whereas simple templating arguments favour the (l0 0001$)$ face. The energies of the two interfaces are very similar, so purely energetic arguments cannot 
(a)

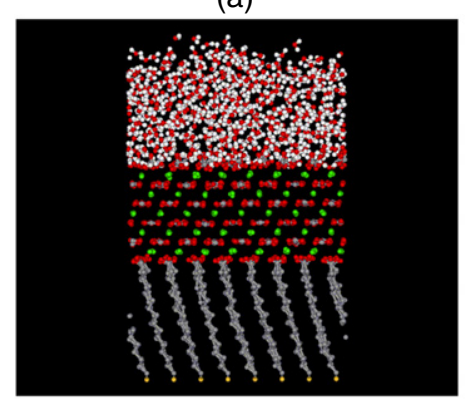

(b)

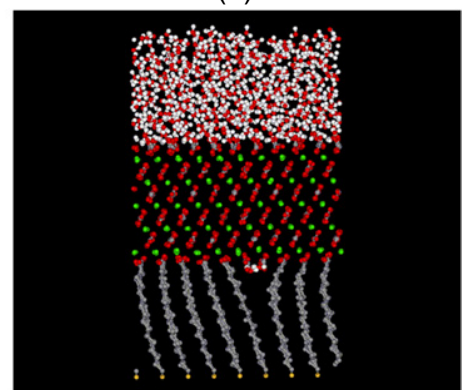

Figure 1. Calcite crystals nucleating on top of alkylthiols (figure supplied courtesy of Dr D M Duffy). Above the calcite is a supersaturated solution of calcium carbonate. The substrate comprises alkylthiols with carboxylic acid head groups; these molecules have been self-assembled on what, in an experiment, would be a gold substrate. The calcite crystals (the central layer) show (figure 1(a)) excellent matching of the $\left(\begin{array}{llll}0 & 0 & 0 & 1\end{array}\right)$ face. What is observed (figure $\left.1(b)\right)$, however, is the $\left(\begin{array}{lll}0 & 1 & 1\end{array}\right)$ face. To favour the observed face, one needs to insert bicarbonate ions to increase the charge density of the monolayer in the $\left(\begin{array}{llll}0 & 1 & 1 & 2\end{array}\right)$ interface and to cancel the long-range dipole of the polar interface. Simple templating arguments are inadequate.

explain the face selectivity. To favour the observed face, it proves essential to cancel the longrange dipole of the polar interface by inserting bicarbonate ions to increase the charge density of the monolayer in the $\left(\begin{array}{llll}0 & 1 & \overline{1} & 2\end{array}\right)$ interface. Figure 1 demonstrates the excellent matching

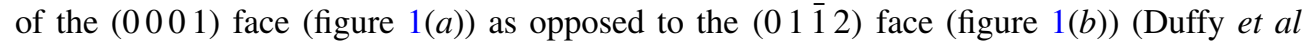
2005, Duffy and Harding 2005a, 2005b). However the $\left(\begin{array}{ll}0 & 1 \\ 1 & 2\end{array}\right)$ interface is favoured by the geometric BFDH model of crystal growth (Friedel 1907, Bravais A. 1913, Donnay and Harker 1937) as it has a larger interplanar spacing. Overall, the mechanisms involved in selective crystal nucleation on organic substrates are poorly understood. Templating is often cited as the dominating mechanism, but supporting experimental evidence is sparse.

\section{Organization at the mesoscopic scale}

\subsection{Qualitative aspects of organized structures}

At the mesoscale, organized structures differ significantly in appearance, as well as in their varied origins. Novel chemical approaches yield varied nanostructures (Ozin and Arsenault 2005, Antonietti and Ozin 2004). Sometimes one can identify a central order parameter that underpins quantitative description, but there may be no unique definition that allows comparisons between different structures. Novel approaches by mathematicians (e.g. Müller's work on Young measures, http://www.mis.mpg.de/sm/microstr/microstr-jahrbuch.html; see, e.g. Kohn and Müller (1994)) are already giving insights into understanding microstructures and may well lead to new ways to analyse organization. The general issues of robustness (sensitivity to initial conditions) and selectivity (one can choose an intended structure by external constraints or minor changes in molecular structures) will be discussed later. There are perhaps five main features that might be used to compare one example with another, as well as a number of subsidiary characteristics.

Dimensionality. In how many dimensions is the structure ordered? Convection cells can show only two-dimensional ordering, although their periodicity is often related to the depth of liquid. Phase separation on passing through a phase boundary may lead to two-dimensional 
structures. Particles confined to a track or ledge may order in just one dimension. Other structures can be fully three-dimensional over many periods in each dimension, e.g. the longer period void lattices.

Periodic or aperiodic order. A crystalline form of whatever dimensionality is easily recognized both in real space and by diffraction. Periodic regular arrays are common at the mesoscale, as are systematic deviations from regularity. Examples of periodic arrays include those derived from elastic interactions (which may link a scale length of one some sort with another periodicity length), domains from competition between terms, the fluxoid lattice of superconductors (again with competition between terms), instabilities such as spinodal decomposition or convection cells and geometries of purely electronic origin (Fermi surface geometry, charge density waves). There is usually a clear natural length scale. Other structures are clearly ordered, such as Fibonacci structures or Liesegang patterns, and it is less obvious whether there is a characteristic length. Fibonacci structures are seen both in natural structures, such as sea shells, and in man-made ones, such as the spiral patterns formed from assemblies of $10 \mu \mathrm{m}$ particles with an Ag core and a silica coating (Cao et al 2005). Liesegang rings (Liesegang 1896, Hedges 1932, Stern 1954) can be produced in precipitation reactions, when periods of significant and weak precipitation alternate. There is still a debate about the importance of factors such as supersaturation, coagulation, adsorption and autocatalysis.

Symmetry or asymmetry. Chiral systems (left- and right-handed forms, see section 3.4) are important and form one class of the common phenomenon of asymmetry. Biologically important molecules are often chiral, although chirality is by no means a special feature of the living. In the case of fluid distribution networks (including natural ones, such as blood vessels, bronchial trees, botanical trees and river basins) asymmetry can arise from general principles such as minimization of power requirements (Gosselin and Bejan (2005) cite Mauroy et al (2004), Weibel 2000, Phillips and Kaye 1997). The exceptions to an established chirality can be puzzling. Left- and right-handed people are both quite common, even if left-handed people are a minority. Rare examples of 'wrong' symmetry tend to be reported in the popular literature, rather than technical journals, e.g. the catching of an apparently unique 'left-handed whelk,' one that spiralled in the opposite direction to the many millions that spiral the other way (Parsons 1968), Gould (1993) cites a similar example for a periwinkle. Ueshima and Asami (2003) note that the mirror image form of a snail's body plan is determined by a single gene, and genital mismatch can lead to establishing chiral speciation. This they observe for the Japanese land snail, Euhadra.

Texture. Texture is best defined by example. Some mesostructures show small objects ordered yet widely spaced (e.g. the first observations of void lattices in irradiated Mo, shear planes in a non-stoichiometric oxide, colloids in solution). Other examples show fuzzy structures with variations of some sort over each unit (spinodal decomposition, some examples linked to convection cells, short period bubble lattices in irradiated metals). Still further examples are domain structures, where there are fairly sharp boundaries between regions. There is clearly a subjective component here, but no one would mistake the early void lattice in Mo (Evans 1971) for the standard spinodal decomposition.

Accuracy and contrast. Are the ordered units clear or is the order poorly resolved? For periodic structures, can one see 'defects', such as dislocations or missing units (vacancies)? 
(a)

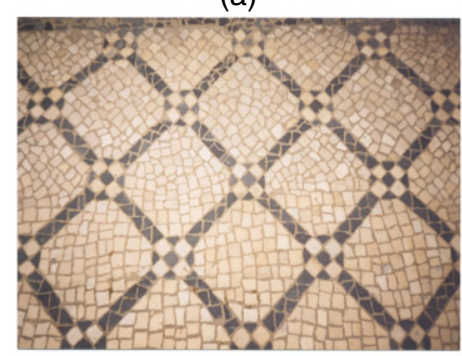

(b)

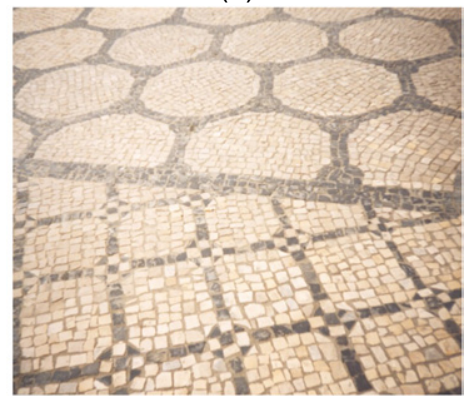

Figure 2. Pavements in Aveiro, Portugal, made of tessellae (photo by A M Stoneham). (a) The periodic structure is a programmed organization, i.e. designed to various degrees by a central planner and by the craftsman putting the tesselae in place. Yet the content of each square (and also the black divisions, especially to the top and centre) show a fair degree of randomness consistent with short-range order. There is also a substrate constraint that runs roughly horizontally in the middle of the figure. (b) An interface between two distinct periodic structures. There is a clearly programmed organization. The craftsman has adapted designs to local constraints. The randomness is consistent with short-range order.

Are the units very clearly resolved or only seen with the eye of love? Order can occur to an almost continuous continuous degree and is not simply present or absent. Moreover, accuracy in bio-organization relates to accuracy in performance, not to accuracy of periodicity or shape: DNA must be accurate in those places where decisions are crucial but can contain plenty of irrelevant, useless sections. Our scientific intuitions, when honed on inorganic systems, tend to concentrate on perceived order. It is a human characteristic to identify patterns or even invent them, as in the traditional constellations of stars (private communication, Professor John Harding) and patterns. Tessellated pavements in Aveiro, Portugal, show some of these features of order and disorder (figure 2). The main periodic structure is 'programmed' organization, i.e. designed to various degrees by a central planner and by the craftsman putting the tessellae in place. However, the content of each square (and also the black divisions, especially to the top, centre) shows a fair degree of randomness consistent with short-range order. There is also a substrate constraint that runs roughly horizontally in the middle of the figure. Accuracy and contrast have different meanings, depending on the features sought.

Ordered structures - at least 3D ones — tend to be rather inaccurate. Even weak emerging structures can be rendered visible by diffraction, using reciprocal space rather than real space images. Surface structures remain visible in low-energy electron diffraction even when there is very substantial disorder. In 2D, Shevchenka et al (2006) show various phases formed from binary nanoparticle lattices. In such systems (various combinations of $\mathrm{PbSe}, \mathrm{Au}$, $\gamma-\mathrm{Fe}_{2} \mathrm{O}_{3}, \mathrm{LaF}_{3}$ on a- $\mathrm{SiO}_{2}$ or a-C) superlattices are formed by self-organization, exploiting known interactions between the particles. The quality of the ordering looks reasonable; whether structures would be as good in 3D is hard to know.

Diffraction methods, developed particularly by Hilliard and de Fontaine and their collaborators (de Fontaine and Cook 1970, Cook and de Fontaine 1971, de Fontaine 1973, Cahn 1968a, 1968b), are a sensitive probe of ordering and show very clearly how order emerges. Their work has covered mechanisms such as spinodal decomposition and clustering. Electron diffraction by void and bubble lattices (Sass and Eyre 1973, Eyre and Bartlett 1973) has given results beyond real-space observations. Order was identified when feature dimensions were too small for direct microscopy and where the ordering was incomplete. Diffraction gives 
accurate measures of the lattice parameters of ordered structures, but no useful guidance of shapes of the voids or the bubbles from spot intensities. The emergence of a void lattice from a 'hard-sphere' gas of voids has been noted: a ring of intensity is observed at first, evolving into spots as the lattice evolves. In some cases there are signs of extra, unexpected, spots appearing in diffraction from a void or a bubble lattice. The origin of these spots is not clear. Reciprocal-space theory can also prove effective (e.g. Stoneham (1971) and by Tewary and Bullough (1972) on the void lattice) because the total energy can be written concisely in terms of the Fourier transforms of concentration fluctuations and of atomic displacements. Such energy expressions yield stability conditions for ordering and indications of how and how fast an instability may develop.

The range of the order: is there any evidence of long-range order? Even hard spheres will show local order. Short-range order may occur through competitive capture of a limited material resource (which might be the atomic species that makes a precipitate or vacancies creating a void). Long-range order is implied when crystal axes remain the same for large distances (well-defined edge dislocations that modify this tend to be evidence of, rather than against, long-range interactions). In liquid phases, the organization of colloids or the like, is frequently described as 'self-organized,' but usually seems to result from shorter-ranged interactions. Many examples are known for the ordering of biomolecules, e.g. actin fibre bundles (De Rosier et al 1980) or tobacco mosaic virus, and the presence of long-range interactions is now generally accepted (Millman et al (1984), Parsegian and Brenner (1976), see section 3.4.2).

In living systems, where organization can take impressively varied forms (e.g., Bejan 2000), most examples either involve only a relatively small number of repeat units or show short-range (rather than long-range) order. The review by Naik and Stone (2005) mentions the scales covering infrared-sensitive organs of pit vipers and pythons (Campbell et al 1999), insect cuticle systems (Parker et al 2000) and the setae on gecko feet ((Autumn et al (2002); there are credible reasons to doubt the now accepted view of van der Waals as the whole explanation of geckos being able to walk over ceilings) and the structures leading to hydrophobicity of lotus leaves (Barthlott and Neinhuis 1997, Cassie and Baxter 1944, Wentzel 1936, Cheng and Rodak 2005). Biomineral structures can show longer-range periodicities and might be more directly comparable to the inorganic systems already noted.

\subsection{Bringing order to organization}

What are the likely culprits in persuading a system to organize? Seul and Andelman (1995) have looked at order parameters and at the nature of the competing energy terms for a number of systems. They identify similarities and differences in a number of examples, including normal and superconducting regions in superconductors, ripple phases in a vessicle, Turing patterns of chemical reactions, Rayleigh-Bénard convection cells, domains and bubble phases in magnets, and stripe and bubble domain phases in 2D and 3D organic systems. Whilst their approach is somewhat different from the present one, the two analyses have some key ideas in common.

Some organized structures have a natural origin at the atomistic level, such as crystal structures with non-random orientations and surface facets. These may include shaped crystals (spherical, discs, rods, etc); even tetrapods can be grown at the nanoscale. Vernier or moiré structures can arise at any length scale. Special non-periodic structures (spirals, Fibonacci orderings) are often seen in nature, but do not seem to need any special 'clever' natural techniques, such as those exhibited by DNA. There can be systematic composition fluctuations 
(e.g. selective precipitation) in liquids as well as in solids. In such cases, the ions are usually relatively mobile.

Electrostatic forces are generally important, even when screened. The relatively slow distance dependence $(1 / r$ for the unscreened interaction) means that electrostatic interactions provide relatively small forces but relatively large binding energies (think of a repulsive $r^{-N}$ interaction and an attractive $r^{-1}$ interaction; when in equilibrium, the forces $-\mathrm{d} E / \mathrm{d} r$ are equal and opposite, which means the longer-range interaction energy is larger than the shorter-range energy by a factor $N$ ). Other orderings ultimately electrostatically driven include charged species responding to dielectric constant variations or to polarizability variations if there is an electromagnetic field.

Even weaker short-range interactions can be very important, and this underlies some of the principles in natural structural materials. For instance, for a strong adhesive, it is usually not the energy by which a polymeric adhesive molecule sticks to a substrate that matters, provided it is big enough: the work done in pulling two pieces apart is mainly used to break very many small bonds as polymer molecules are pulled through the network (e.g. de Gennes 1990). A distinct but analogous situation occurs for some sea shells and synthetic sea shells. They need to be strong (rigid), hard (resistant to indentation) and tough (needs a lot of energy to break them up). This is achieved using a 'bricks and mortar' structure, with calcium carbonate 'bricks' and protein 'mortar' (Rubner (2003) commenting on Tang et al (2003)). Tang et al mimic the shell structure using as bricks clay platelets, negatively charged and a few nanometres thick. Their mortar was a polymer, positively charged. Under deformation, the relatively soft electrostatic bonds readily allow the polymers to deform and rearrange; in so doing, a lot of deformation energy is used, so the material is tough.

There can be competing roles for hydrophobic and hydrophilic regions of surfaces. The consequences can be counter-intuitive. Thus, Aranda-Espinoza et al (1999) discuss the repulsion between positively charged vesicles (typically $20 \mu \mathrm{m}$ across, with a Debye screening length of order 1-10 nm) and the negatively charged surfaces of colloids, where the interactions affect self-organization. In consequence, there were separate macroscopic adhesive and non-adhesive zones. The mobile counter ions were important, with an interplay between the entropic and electrostatic interactions. The consequence is that adhesion in one region of the membrane can affect the adhesion hundreds of screening lengths away. Nor should one ignore much simpler phenomena, since even the local $\mathrm{pH}$ might be a control parameter. A report in The Sunday Times (14 March 1999) observed that coral reefs could be re-grown and that limestone building materials could be created by exploiting electrochemistry, simply making the water locally a little more alkaline.

\subsection{Organization mechanisms}

One possible classification of organizational behaviour focuses on mechanism. One general principle is that energy minimization (global or local) determines structure. However, this is not always the underlying principle. Some structures are geometric in origin. Even at the mesoscale or the macroscale, one can have Vernier or moiré patterns, or features associated with wave diffraction (such as sand or pebble structures). Further, many systems, especially living systems, are not in an equilibrium state. Whilst structures can sometimes be understood as en route to a minimum energy conformation, it will be common to find quasi-steady states or other dynamically determined states. Kinetic control can have several forms. One corresponds to the fastest-forming instability, as in convection cells or spinodal decomposition. Another is diffusion related, e.g. some eutectoid structures or Liesegang phenomena (see, e.g. Kahlweit 
1965, Klue and Mullins 1969, van Roijen et al 1975, Kai et al (1982), Dee (1986), Phys. Today (Jan 1999) p 20 on Zik et al (1998)). The Turing wave model shows some parallels (Turing 1952, also pp 123-125 of Smith (1968)), invoking non-linearities in rate equations. Even systems as dynamic as a sphere dropped into a liquid show an apparently organized splash pattern (the classic text is Worthington 1908). There are always subtle features: in these splashing processes, the air above the splash, which is not passive, determines whether or not there is the classic corona photographed by Worthington (see also Xu et al (2005), Quéré (2005)).

Optimized free energy. In so far as the structures reflect an optimum energy (stable or metastable structures), it is likely that the major interactions can be grouped in a few ways. Clearly, there are covalent bonds that try to impose specific bond lengths and angles at the atomic level. Volume exclusion due to the exclusion principle also operates at this scale. But, at the larger scale, the key interactions are largely those that can be described by the macroscopic ideas of elastic forces and electrostatic interactions. Of course, a complete description needs further elements: hydrogen bonds and Van derWaals interactions are significant examples. As already noted, slowly varying interactions contribute relatively more to the total energy and rapidly varying interactions to the forces that must be balanced in equilibrium.

Symmetry breaking. An important discussion by Ortoleva (1980) addresses fascinating examples of biopatterning, starting with the remarkable symmetry breaking of the egg of Fucus, apparently an example of a Turing reaction. For Turing reactions, the Belousov-Zhabotinsky (BZ) oscillator and the like, the key issues may be diffusion (e.g. fast species that inhibit reactions and slow species that autocatalyse it), and the description will normally include rate equations with some feedback. For the BZ chemical oscillator and the CIMA reaction and so-called chicken skin patterns, see (Turing 1952, Scott 1995, Winfree 1991, Ouyang and Swinney 1991, Winfree 1974, Winfree 1980, Perez-Munuzuri et al 1991). Kitahata and Yoshikawa (2005) discuss the additional role of a photon flux. Prigogine's (1980) wideranging discussion of the evolution of non-equilibrium systems discusses the related Marangoni instability ('roll cells' p 152 of his book) and the BZ chemical oscillator.

Kinetic pattern formation. Pattern formation is rather general in supersaturated material systems, when structures are often selected through kinetic reasons (Kirkaldy 1992). The phenomena range through diffusive-capillarity surface instabilities (Wagner 1954, 1956, Chandrasekhar 1961, Mullins and Sekerka 1964), spinodal decomposition and Ostwald ripening (Cahn 1961, 1968a and 1968b, Hillert 1965), free dendrites and Widmanstätten figures, eutectics, eutectoids, discontinuous precipitation and similar patterns, cellular solidification and convection cells, forced velocity solidification, Liesegang phenomena and wave-number selection in first-order phase transitions. Other examples may well be the droplet and the stripe patterns that emerge when $\mathrm{Pb}$ is deposited on a clean $\mathrm{Cu}$ surface (Plass et al 2001).

Kirkaldy asks to what extent is the principle of minimum dissipation (and the related linear principle of most rapidly fastest-growing wave-numbers) the main part of an explanation. How is mode selection (e.g. between lamellar and rod decomposition) achieved? What role do fluctuations play? To what extent should one invoke ideas such as scaling laws, universality classes, deterministic chaos and the wealth of models that mimic behaviour (cellular automata, fractals, self-organized criticality)? The possibility is emphasized-rightly, I believe-that some of the same mechanisms that underly microstructure formation in steels may also underpin the growth and stabilization of patterns in the biology. 
Diffusion control. Smith (1998) observes that genes are switched on or off by morphogen concentrations being in particular ranges, and these concentrations are controlled by diffusion. He also makes the interesting point that for both elastic and contractile protein fibres, both elasticity and contractility depend on calcium concentrations. Braterman and Cairns-Smith (1987) have argued that banded iron formations (chemical sedimentary rocks with alternate iron rich and iron poor layers) can be understood by abiotic photo-oxidation, with iron supply as the key limiting factor.

Colloid systems and plastic sphere analogues in ionic solutions. In this rich field of study, experiments often concentrate on model systems, such as plastic spheres in a liquid, that can be tailored to mimic the behaviour of natural colloidal systems. Thus, one finds the spontaneous ordering (including bimodal forms) of nanoscopic gold clusters (Kiely et al 1998), periodic colloid structures involving electrostatic interactions (a fairly comprehensive review by Efremov 1976), ordered colloid states for which Van der Waals forces are significant (van Megen and Snook 1976) and evidence of attractive and repulsive dispersion forces between macromolecules in solution (Gengel 1978; see also Adair and Suvaci 2000, Leubner 2000). Other systems that have features in common include ordered water and the ultrastructure of the cellular plasma membrane (Schultze and Asunmaa 1970) and the influence of shear on crystallization (Ackerson and Clark 1984). There are clear analogies with ordering in biological systems, such as the tobacco mosaic virus (Millman et al 1984, Parsegian and Brenner 1976).

\subsection{Characteristic features of periodic structures}

In this section, we shall follow the classification of Stoneham (1975), who analysed many of the periodic structures in inorganic systems and identified a number of categories of regular arrays with periodicity on an intermediate scale. Another interesting scheme has been given by Muthukumar et al (1997), who identified what they described as three principles. Their first principle is based on competing interactions and sequences, which they illustrate with a chain with four types of domain, each with some functionality. Different sequences of domains lead to different degrees of stability. Their second principle is based on entropic frustration and topological dereliction, emphasizing configurational entropy issues, and the topology of free energy landscape, which may favour structures less favoured by the first principle. Their third principle concerns the spontaneous selection of primary length scales and symmetries, especially when there are competing interactions. This leads to questions as to how scale lengths might be tuned.

3.4.1. Cases involving a single dominant interaction. In the cases for which just one interaction dominates, it is surprising that there is an energy minimum at all, since many interactions depend monotonically as a function of spacing. In the case of the elastic interaction, theory can indeed show energy minima as a function of spacing: a long-ranged, weakly attractive interaction, with short-range terms being repulsive at distances related to some structural feature of the ordering units.

For periodic structures, the energy per unit can be written down exactly in some reference cases. The ordering of shear planes in non-stoichiometric oxides (Stoneham and Durham 1973) is an example. The analysis, following Stoneham (1971), has some important general features. Imagine a periodic array of defects in an otherwise perfect elastic lattice (the generalization to a polarizable lattice is straightforward). The defects might be precipitates or voids or planar defects like shear planes. We may represent these defects by defect forces; these forces $\boldsymbol{F}(\boldsymbol{R})$ which, if applied to the atoms or the ions of the perfect solid, would lead to the same 
displacements as the actual defects do. In the periodic array, we need the Fourier transform of the defect forces, $\boldsymbol{F}(\boldsymbol{q})$. There is also a lattice response function, usually the elastic Green's function $\boldsymbol{G}(\boldsymbol{q})$, which is essentially an inverse force constant and which relates displacements to the forces. The reciprocal-space form of the Green's function $\boldsymbol{G}(\boldsymbol{q})$ is known analytically for important classes of system, e.g. cubic lattices, whereas its real space form is far less convenient. The elastic energy of a regular array of these force units is the sum of the elastic energy of deformation reduced by the work done by the defect forces in driving the deformation. This is given by a sum $\frac{1}{2} \Sigma_{q} \boldsymbol{F}(\boldsymbol{q})^{*} \boldsymbol{G} \cdot(\boldsymbol{q}) \cdot \boldsymbol{F}(\boldsymbol{q})$, where the sum is over the wave vectors corresponding to the super lattice wave vectors, i.e. a finite sum readily evaluated by computer. The expression is then exact within elasticity theory, though not, of course, exact in every respect. For example, $\boldsymbol{G}(\boldsymbol{q})$ will usually assume no phonon dispersion, nor will it include the effect of the voids on the elastic properties (see below); moreover, the defect forces $\boldsymbol{F}(\boldsymbol{R})$ may not be known with any certainty.

The proposal by Stoneham and Durham that shear plane ordering was associated with elastic and electrostatic interactions gained support from a number of later studies (Catlow 1979, Cormack et al 1982, Tilley 1977, Iguchi and Tilley 1977). Not only does this theory suggest reasonable values for shear plan spacings but it also explains why, in a cluster of planes, the spacings between the outer planes are larger than those at the centre of the cluster, as observed. In the case of the void lattice and the related bubble lattices [1], there is still some controversy, so these systems will be treated separately in section 3.4.5.

On smaller scales, strain energy would seem to be a significant component for interstitial alloys, e.g. $\mathrm{V}_{16} \mathrm{~N}, \mathrm{Ta}_{64} \mathrm{C}$, for ordered vacancy structures, e.g. $\mathrm{V}_{6} \mathrm{C}_{5}$ and related systems and for ordered precipitates (Khachaturyan 1968, 1978, Tewary 1973a). Possibly the ordering of oxide precipitates in ion-implanted silicon is of this type (van Ommen et al 1986). Almost certainly the staging of graphite intercalation compounds involves these elastic interactions.

Mechanisms of this class need a long-range attractive interaction (since a mesoscale periodicity is needed) but with some shorter-range repulsive term that leads to a minimum energy at finite spacing. The short-range term must be related to structural detail in some way, which is why the result is a spacing that is related to some other length scale, such as the structure within the individual shear planes.

3.4.2. Domain structures, where periodicity can result from competition between two independent energy terms. When the short- and long-range terms are independent, there are more opportunities to form varied mesoscale structures. Ferromagnetic and ferroelectric domain structures demonstrate widely varying periodic and ordered forms. In the case of nearly isotropic ferromagnets, the characteristic domain size $D$ reflects a balance struck between the domain wall energy per unit volume $\sim D^{-1}$ and the demagnetizing field energy $\sim D^{+1}$. For a highly anisotropic ferromagnet, it may be a compromise between the domain wall energy $\sim D^{-1}$ and the anisotropy energy $\sim D^{+1}$. Ferroelectrics show similar competing energies, with also elastic interactions associated with the domain wall dislocations. In a more general sense, Muthukumar et al (1997) emphasize the role of competing interactions and sequences in a chain with four types of domain, each with some functionality, such that the different degrees of stability become associated with different sequences of domains.

The fluxoid lattice in type II superconductors in a magnetic field likewise shows competing energy terms (Traube and Essmann 1966, 1968), with mesoscale structures for filaments of normal metal in a superconducting matrix. If the penetration depth is much greater than the coherence length, there is a negative energy associated with the boundaries between the normal and the superconducting regions. The detailed configuration is a compromise between this, the magnetic field terms and a repulsion between the flux lines. 
The ordering of tobacco mosaic virus was reported by Bernal and Fankuchen (1941). Parsegian and Brenner (1976) noting that this was similar to other observed apparently self-organized biological structures (muscle sarcomeres, the visual cornea and the gel of sickle cell haemoglobin) tested the 1941 data to see if it could be explained as a competition between attractive van der Waals interactions and electrostatic repulsion. Yet their analysis suggested that the data were not consistent with a force balance model. However, subsequent experiments and analysis (Millman et al 1984) came to the opposite conclusion: a balance between electrostatic and van der Waals forces did indeed explain the stability of the tobacco mosaic virus gels near physiological conditions and was a good first approximation in other cases.

Charged colloids at water interfaces have repulsive Coulomb interactions, yet an attractive capillary part that can dominate at large separations (Oettel et al 2005). There is an energy minimum at intermediate separations when the colloid radius and the Debye screening lengths are comparable. Of course, there can be interactions that interfere with ordering at surfaces. Thus organic films on liquid $\mathrm{Hg}$ show a range of interactions: intermolecular van der Waals interactions promote self-assembly and both short- and long-range order, whereas the end group structures can favour a less ordered form (Magnussen et al 1996). Of course, the organization that results can be short-range only. Thus, when III-V quantum dots capped with an organic-like octanethiol are put on a surface and evaporation allowed, there is rather nice short-range order, but rather little long-range order. Dynamically arrested glassy states can arise in three dimensions as well (Stradner et al 2004), with implications for protein and DNA self-assembly. Liu et al (2006) observe wave-like structures in microtube bundles, apparently selected by a balance between the bending energy of the bundles and the elastic energy of the surrounding network. This, again, would seem to be a balance between two energy terms. These patterns are seen in living organisms, such as frog's eggs and fruit-fly cells, but there appears to be no genetically related process directly involved.

Many organized structures common on surfaces are likely to involve competition between two terms, e.g. an attractive elastic term (e.g., Stoneham 1977) and a repulsive dipole-dipole interaction. Anthraquinone molecules self-assemble on a $\mathrm{Cu}\left(\begin{array}{lll}1 & 1 & 1\end{array}\right)$ surface into a large twodimensional honeycomb network with pore diameters of $50 \AA$, about five times larger than the size of the constituent molecules (Pawin et al 2006). There seems to be balance between substrate-mediated long-range repulsion and intermolecular attraction, the attraction involving hydrogen bonding between a carbonyl oxygen and an aromatic hydrogen. Water clusters, each comprising six molecules centred above a fluorine, form on $\mathrm{CaF}_{2}$ (Lehmann et al 1994), though the precise forces are not certain.

3.4.3. Ordering associated with electronic structure. One of the simplest descriptions of the electronic band structure of a solid is the nearly free electron model. The electronic density of states has the standard form for free electrons, usually scaled by an effective mass, but is modified at zone boundaries. At these zone boundaries, there are important effects from even weak interactions with the periodic part of the potential due to the ionic lattice structure. In particular, a gap opens up. There is extra stability when the electron density is just sufficient to fill the band below the gap. Alternatively, for a given electron density (and Fermi wave vector) a deformation can exist that exploits this energy lowering. This deformation may have a characteristic wavelength related to the Fermi wave vector, and not rationally related to the undeformed lattice structure. This is the case for charge density waves.

Even simple systems can show distortions that are non-commensurate, in the sense that the wave vector $K$ characterizing these distortions is not simply related to the lattice spacing. This is known as the Peierls instability, and is exemplified by charge density waves (Kittel 
1996, Wilson et al 1975, Littlewood and Heine 1981) in systems such as $\mathrm{TaS}_{3}$. For a simple metal, an electron gas filling all conduction band levels to some wave vector $k_{\mathrm{F}}$, deformation potential coupling leads to distortions with wave vector $K=2 k_{\mathrm{F}}$ that lower the sum of the electronic and elastic energies. Suppose there is an elastic strain $\mathrm{A} \cos \left(2 k_{\mathrm{F}} x\right)$, where we consider one dimension only for simplicity. The elastic energy per unit length is $c A^{2} / 4$ if $c$ is the relevant elastic constant of the metal. The effect of the deformation is to provide a perturbation $2 D A \cos \left(2 k_{\mathrm{F}} x\right)$, with $D$ the deformation potential. This perturbation opens up a band gap at the Fermi level, reducing the electronic energy. Straightforward calculations (Kittel 1996, p 301) show that the lowest energy corresponds to a strain amplitude $A \sim 4(W / D) \exp [-1 / N(0) V]$, in which $W$ is the bandwidth, $N(0)$ the density of orbitals at the Fermi level and $V=2 D^{2} / c$ is an effective electron-electron interaction.

Charge density waves illustrate the general principle that structures favoured are those that ensure that the electrons can just fill a band. The principle has wide application, e.g. to long-period alloys, or in determining the relative stabilities of intermetallic phases (Carlsson and Meschter 1989) in the Stoner criterion for ferromagnetism. For very thin metallic films, the quantization of wave vectors perpendicular to the film is important. In particular, as the film grows, the energies of particular one-electron states cross the Fermi level (Wei and Chou 2002), and alter the density of states so that particular thicknesses are stabilized. This mechanism provides some bias to these particular thicknesses in layered structures.

3.4.4. Instability structures. These structures emerge when an unstable initial configuration, such as a supersaturated solution evolves into a periodic structure characteristic of the fastestforming instability (see, e.g. Kirkaldy 1992). In such cases, configurational entropy can matter more than enthalpy. Muthukumar et al (1997) suggest entropic frustration and topological dereliction (meaning configurational entropy; the topology refers to how the free energy landscape may favour structures less favoured by the first principles) are general principles. The examples given here will be discussed in different contexts later.

Spinodal decomposition. (Cahn 1968a and 1968b, Anderson 1972). Where mixed phases can occur, and where $d^{2}$ (free energy)/ $/ d c^{2}$ can be negative, then 'uphill' diffusion can occur, with species moving against the concentration gradient. The driving force is partly surface energy (usually small), partly elastic energy due where phases with different ideal lattice parameters match coherently (and anisotropy energy is important). The phase is unstable against small fluctuations in composition, and new phase(s) form by a continuous process, typically on cooling at fixed composition. Examples include borosilicate glasses that form regions of high and low $\mathrm{SiO}_{2}$, and alloys, such as $\mathrm{Cu} / \mathrm{Ni} / \mathrm{Fe}$ solid solutions. The signs of ordering are usually clear, but most micrographs show rather untidy structures. Cahn's analysis relates the characteristic length scale to the shortest time that characterizes an instable emerging structure, and hence predicts the dependence of this wavelength on composition and on the temperature relative to the temperature for the coherent spinodal.

Convection cells. (Chandrasekhar 1961). The instability needs a minimum temperature gradient (with lighter material at the bottom), when the internal energy release from buoyancy can be steadily dissipated by viscous dissipation. If one seeks a periodic condition such that there is no fall off with time, surface tension may be important. It may be that various premelting and solidification phenomena (Hämäläinen 1967, 1968) are related to convection cells. If a film is melted, then solidified and remelted, segregation may occur associated with convection cells during solidification from the first liquid phase, and this can then affect where the earliest 
melting takes place subsequently. However, as Hämäläinen has noted (private communication to AMS, 1972) the melt structures look different, and the spherical cells can collide elastically with each other; it is possible that the elastically controlled structures may be a better analogy.

Solidification. Cellular and dendritic growth (including eutectic structures) occurs widely in materials from alloys to liquid crystals (e.g. Mullins and Sekerka 1964, Guy 1972, Oswald et al 1991), and involves competition between diffusion, capillary forces associated with interfacial energies and crystal anisotropy. Mullins and Sekerka noted that the movement of the solidification front required the rejection of both latent heat and solute. Locally, the growth velocity is proportional to a concentration gradient. If a bump occurs on the interface, the concentration gradient increases locally, so the growth velocity can rise. In some circumstances, the instability is limited by surface tension, essentially at higher growth velocities; the temperature gradient can be a source of stability at lower velocities. There appear to be systematic dependence of solidification structures on the composition and on $\varphi$, the entropy of fusion in units of the Boltzmann constant $k_{\mathrm{B}}$. If $\varphi \sim 1$, as for metals and some organics, then there will be ordered phases for both components. If $\varphi \sim 3$ or more, one finds lamellae or cylindrical rods, depending on the volume fractions of the resulting phases.

These ideas have many parallels that include spontaneous symmetry breaking with consequences that show order in some sense. Constitutional supercooling is another case. Near a solidification front, growth alters concentrations in the liquid and solid, thus affecting the melting/freezing temperature; this is important when there is a temperature gradient. Low constitutional supercooling gives cellular growth, gradually changing to dendritic growth for high constitutional supercooling. Fingering patterns in combustion are another example (Zik et al 1998). There is then a broad class of diffusion-controlled orderings. Some have very considerable practical importance, such as the eutectic structures in irons and steels. Pearlite (alternate layers of ferrite and $\mathrm{Fe}_{3} \mathrm{C}$ ) is a lamellar eutectic structure that is formed from austenite through interfacial diffusion of $\mathrm{C}$ from the ferrite to the $\mathrm{Fe}_{3} \mathrm{C}$ during the growth. Other diffusion-related phenomena, e.g. Liesegang phenomena, have already been noted.

3.4.5. The void and bubble lattices in irradiated solids. Void and bubble lattices have been observed in a wide range of systems, especially neutron-irradiated or He-implanted. The first observation (Evans 1971) was in Mo, neutron irradiated to some 50 displacements per atom. Evans observed several striking features. First, there was a body centred cubic (bcc) lattice of voids, each void being about $2 \mathrm{~nm}$ across and separated by about $20 \mathrm{~nm}$. Secondly, this was a 3D lattice, with axes parallel to those of the underlying Mo crystal. The void lattice included tens of millions of voids. Thirdly, the voids were arranged with great regularity, although the individual voids appeared to vary in appearance. Fourthly, the void lattice contains very clear edge dislocations. Subsequent observations showed that void lattices occurred in bcc and face centred cubic (fcc) metals, with the same structure as the underlying atomic lattice. The void spacings varied over quite a range, with lattice spacings up to $\sim 140 \mathrm{~nm}$ (figure 3 ). Voids arranged on a lattice were only seen under suitable conditions of irradiation and temperature. When a lattice was seen, the void spacings were usually similar to the typical sizes and spacings of the disordered voids under somewhat similar conditions (Moteff et al 1974).

Subsequently, so-called bubble lattices were observed following He irradiation (Johnson and Mazey 1979, Johnson et al 1990). The actual pressure in these bubble lattices is significant, but the description as a 'bubble' should be considered a metaphor. These lattices differ from void lattices in several respects. The lattice parameters ranged mainly from about 5 to $8 \mathrm{~nm}$, with diameters mainly $\sim 2 \mathrm{~nm}$, with no systematic size-spacing trend discernable (figure 4 ). 


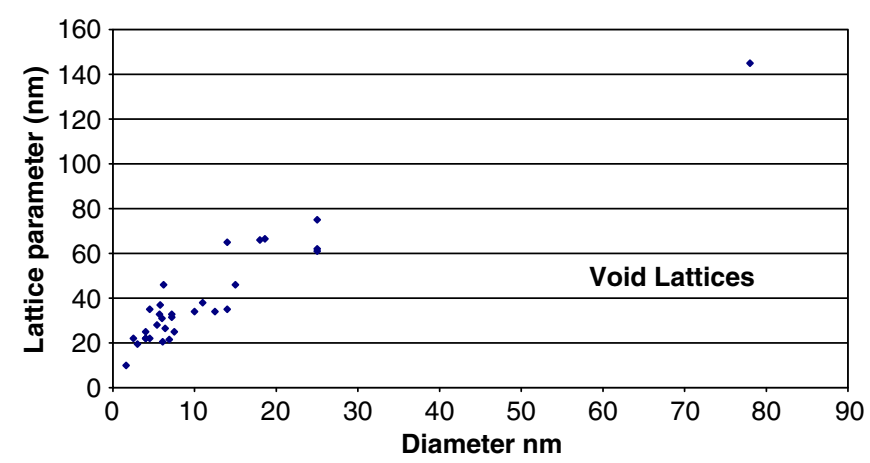

Figure 3. Void lattice spacings as a function of void diameter. The data have been taken from values tabulated by Ghoniem et al 2002.

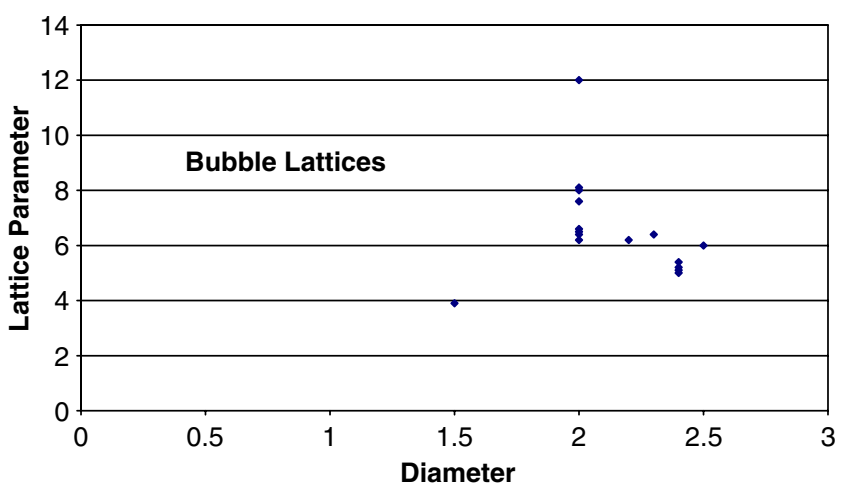

Figure 4. Bubble lattice spacings as a function of bubble diameter. The data have been taken from values tabulated by Ghoniem et al 2002.

The bubble lattices seem far less tidy than the long-period void lattices, and periodicity may be clear only in diffraction experiments. Likewise, periodic arrays of dislocation loops have been seen in $\mathrm{Cu}$, proton-irradiated to 2 displacements per atom (Jäger et al 1987, 1988). Domains are seen, each with perhaps 5-10 layers with spacing of order $100 \mathrm{~nm}$.

The 'void lattices' reported in $\mathrm{CaF}_{2}$ under electron irradiation (Johnson 1977, Chadderton et al 1976, Murr 1974) appear to comprise fluorine vacancies (so they are effectively calcium colloids). The ionic nature of these systems raises further issues (see, e.g. Hayes and Stoneham 1985, Itoh and Stoneham 2001) that have yet to be analysed in detail.

There is a question as to whether the same mechanisms underlie void and bubble lattice formation. My personal view is that the dominant ordering mechanisms differ for short-period (say 1-2 nm) bubble lattice and for those original long-period void lattices, with $\geqslant 20 \mathrm{~nm}$ spacings and their striking edge dislocations. I have reviewed a number of possible explanations of void lattices (Stoneham 1974, 1975). Some of the explanations were clearly inadequate, so I comment mainly on the more plausible explanations here. They divide themselves into two main classes: those based on energies, and primarily elastic energies, and those based on kinetics. 

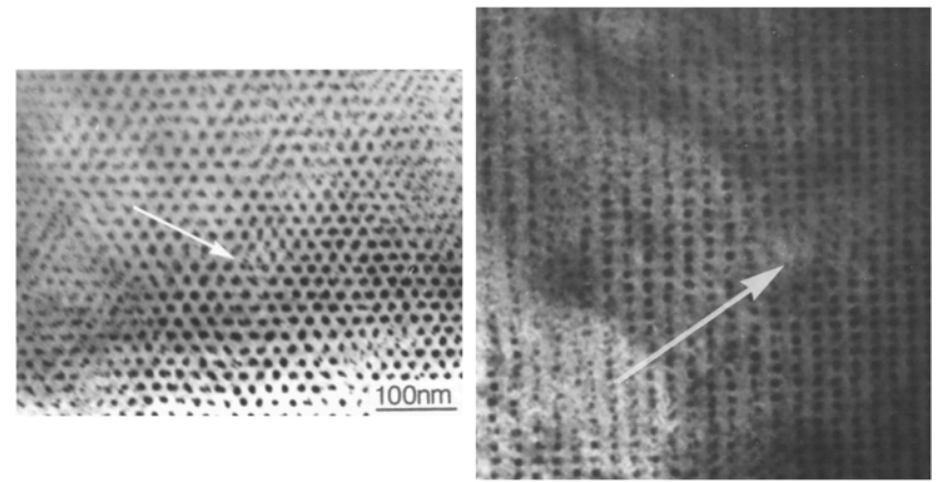

Figure 5. Void lattices viewed from different directions in Mo. The arrays were produced by $2 \mathrm{MeV} \mathrm{N} \mathrm{N}^{+}$ion irradiation at $870^{\circ} \mathrm{C}$ (micrographs courtesy Dr J H Evans). The arrows show the cores of an edge dislocations. For other figures showing edge dislocations, see Evans $(1971,1990)$.

The experimental scene. The void lattice has been observed in bcc systems (Mo, Ta, Nb, $\mathrm{W}$ ), fcc systems $(\mathrm{Ni}, \mathrm{Al})$ and certain alloys (e.g. TZM and $\mathrm{Mo}_{0.95} \mathrm{Ti}_{0.05}$; for complete reference see Ghoniem et al 2002). High stacking-fault energies (e.g. Mo, Ni) seem to favour a void lattice. It is produced after neutron or ion bombardment, but not yet under electron irradiation. The void lattice has the same structure and axes as the host, e.g. bcc in bcc hosts, fcc in fcc hosts. Void spacings depend on irradiation conditions; just as for isolated voids, temperature, dose rate and presumably impurities affect spacings. The spacings of the larger void lattices vary roughly monotonically with void diameters (figure 3, based on data compiled by Ghoniem et al), being typically 2.5-3 times the void diameter. For the closer-spaced bubble lattices, there is little evidence of a systematic trend: most bubble diameters are $\sim 2 \mathrm{~nm}$, and lattice parameters are in the $5-8 \mathrm{~nm}$ range.

Void lattices are very stable, both against radiation and annealing. Once formed, the void lattice seems to saturate, without further growth. Whereas isolated voids in Mo shrink at about $1100^{\circ} \mathrm{C}$, and whereas coarsening causes large voids to grow at the expense of smaller ones, those voids in a void lattice show neither feature. Only when the temperature rises above $1500^{\circ} \mathrm{C}$ are there major changes, as groups of voids evaporate.

Even the best observed void lattices show several classes of defect. First, edge dislocations in the void lattice (Evans 1971, 1990, figure 5), with an extra plane of voids present, have been seen in $\mathrm{Mo}$ and $\mathrm{Mo}_{0.95} \mathrm{Ti}_{0.05}$. Secondly, there can be vacant sites on the void lattice. Such cases are common in poorly formed lattices. Thirdly, voids do not seem to be identical, but vary in size and shape from one site to the next. Individual voids are not spherical, nor symmetrically faceted like some larger isolated voids. The precise shape must depend in part on the presence of gas in the void. Fourthly, voids may show small random displacements from the expected lattice site. For He bubbles in Mo, the random displacements are as much as $20 \%$ of the mean separation. Lattices with large spacings tend to be more precise. Finally, some micrographs appear to show several voids on one site; the voids in such groups at one site are usually small.

These data raise three important questions. First, why does this exotic structure occur at all? Secondly, why is it stable against annealing and under further irradiation? Thirdly, can we understand ordered structure's evolution and kinetics?

Kinetics explanations. Mechanisms in which kinetics is the major factor have been popular in recent years, despite several serious concerns. The natural analogies to make are either with coarsening or with spinodal decomposition, though clearly continued irradiation changes 
vacancy numbers and so corresponds to changing concentrations in the alloy problem. Evans has shown that a specimen irradiated at low temperatures can form a lattice on annealing, so one can assume that the important issue is the degree of damage, rather than its rate. In several ways, void lattice formation resembles coarsening, rather than spinodal decomposition. The main features of coarsening are shown by Ardell, Nicholson and Eshelby's (1966) study of aged $\mathrm{Ni}-\mathrm{Al}$, in which elastic interactions between $\gamma^{\prime}$ particles select favourably situated particles for growth, rather than dissolution. Nucleation of precipitates ceases at a very early stage. The growth of larger particles at the expense of small ones is diffusion-controlled and surfaceenergy driven. The elastic interaction between precipitates drives precipitate ordering, but does not affect the kinetics of coarsening, nor does it inhibit diffusion; the interaction merely selects those particles favourably located for growth. All these features (with a slight change of vocabulary and the possible movement of the voids themselves over short distances) fit well with what is known of the void lattice formation, although no corresponding kinetic studies have been made. By contrast, there seems no reasonable analogy with spinodal decomposition. In spinodal decomposition, a new phase forms by a continuous process, where a concentration fluctuation leads to the rapid formation of periodic clusters. The driving force is an interaction energy term which, for the void lattice would require vacancies to tend to congregate even without void formation (i.e. vacancies would tend to move towards regions of higher vacancy concentration when the vacancy concentration gradient is changing sufficiently rapidly).

It is certainly true that the evolution of void, dislocation and point defect populations can be described well by a mesoscopic rate theory (Ghoniem et al 2002, Bullough et al 1975, Doan and Martin 2003) and that spatial instabilities can lead to the evolution of microstructures with characteristic wave vectors (Krishan 1982a, 1982b, 1988). The nature of the evolution involves quite a few factors. Obviously, vacancy and interstitial recombination and diffusion matter. Network dislocations stabilize uniform distributions. Other factors include biases, differences between interstitials and vacancies, including production bias and the biases of interactions with and capture by sinks. Different regimes of behaviour emerge naturally, such as void shrinkage and void growth. The critical wavelength for spatial instabilities (Ghoniem et al 2002 equation (37)), which decreases for higher dislocation network densities (equation (37)), is determined by the defect densities and by the production and sink absorption biases. Systematic trends of average void densities and diameters seem well understood. It is also understandable that voids will show short-range order, since each growing void competes with its neighbours for vacancies. The existence of a predictable fastest-forming wave vector also gives encouragement to the view that ordered voids might be direct analogues of either inclusion structures or of precipitate ordering.

The concerns about kinetics-based mechanisms are serious for the long-period void lattices. For the coarser, short-period bubble lattices, the role of kinetics is more credible. A first concern is that the long-period lattices do not look like the other well-known kineticsdominated periodic structures, which are usually untidy with limited contrast. A second concern comes from the beautiful edge dislocations seen in Evans's pioneering study. It is not hard to understand dislocations in an energy-based model, but very difficult indeed to see how such a neat structure could happen when kinetics control the structure. A third concern is that it is not clear why the void lattices show stability against void growth or loss of lattice order without these structures being close to some free energy minimum that is not part of the simple kinetic theories.

Ideas associated with anisotropic interstitial motion. A quite different idea has been proposed by Foreman (1972), involving neither void-void interactions nor kinetic instabilities. His idea is based on the assumptions that interstitials only propagate along close-packed directions, 
and that these crowdion interstitials have very long mean-free paths. This idea has been taken further in two-dimensional simulations by Evans (2006). Consider a nearly perfect void lattice, with one void displaced from its proper site. Then the flux of crowdions between the void rows will tend to eliminate preferentially the part of the displaced void which is projecting out of line. By qualitative arguments such as these, he argues that the mechanism will tend to tidy up a nearly perfect void lattice by a shadow effect. He also suggests that the shadow effect favours a bcc(fcc) lattice in a bcc(fcc) host. So far, the Foreman mechanism is compatible with other mechanisms involving the void-void interaction; there is no reason why both should not operate at the same time. However, Foreman has suggested that the shadow mechanism explains the evolution of a lattice from a random distribution of voids (although it is not clear how) and is an alternative to the others.

There are substantial objections to this variant of kinetic theory. Whilst it is certainly true that simulations confirm that the actual microstructures that will appear are sensitive to anisotropic diffusion (Ghoniem et al 2002, figure 8, p 29), there seems no evidence that a 3D lattice can originate in this way from a distribution with only short-range order. Moreover, a purely kinetic theory cannot explain the resistance to annealing observed. Nor is there any prediction of the systematic size/spacing ratio. Nor is it clear how dislocations in the void lattice are formed-their subsequent climb might be explained, if ever observed, but their origin is obscure if void-void interactions are neglected. Finally, the interstitials must have remarkable properties. They must be able to pass very close to large imperfections like voids without being diverted into a different close-packed direction, and their mean free path must be at least two void-void separations in the close-packed direction for any shadow effect, and ranges 5-600 seem implausible. This class of theory has supporters, both in the form outlined and in a more complex form (Woo and Frank 1985) involving two classes of interstitial. It is perhaps significant to observe that the so-called void lattice in $\mathrm{CaF}_{2}$ (Johnson 1977, Chadderton et al 1976, Murr 1974) certainly does not form by this mechanism, as the interstitials (H centres,

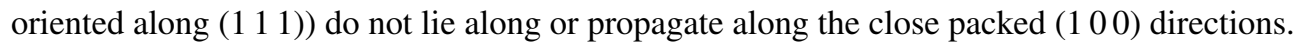

Energetics explanations. Elastic interactions have three essential features: they are common to all crystal structures (bcc, fcc); they can be effective at large spacings of tens of nm and they do not depend on any special electronic properties (like Fermi level structure) that would be modified by the heavy irradiation damage.

Two spheres in an isotropic elastic continuum always attract each other, so the simplest model of spherical voids in an isotropic continuum cannot form a stable lattice. Moreover, the leading elastic energy term, which varies with spacing $r$ as $r^{-3}$, vanishes, so the mechanism of stabilization raises questions. Elasticity-based models must include some degree of anisotropy. The anisotropy can be represented in at least three distinct ways: (i) elastic anisotropy, sometimes generalized to take account of the anisotropic phonon dispersion; (ii) recognition of the underlying host lattice structure, especially the existence of the non-spherical Brillouin zone and (iii) realistic anisotropic void shapes, including faceting and deviations from symmetrical shapes. It may be essential to include the random variations in shape from one void to another.

Malen and Bullough's pioneering discussion (Malen and Bullough 1971, Evans et al 1972) showed how the lattice might be stabilized. Their real space analysis estimated elastic void-void interactions, summing the result over the superlattice. They concentrated on the lowest non-trivial multipole terms in a weakly anisotropic elastic host, finding an interaction proportional to $\delta=\left[\mathrm{c}_{11}-\mathrm{c}_{12}-2 \mathrm{c}_{44}\right]$, which vanishes for elastic isotropy. If $\delta$ changes sign, so does the binding energy of the lattice. For stability, the anisotropy $\delta$ must have the right sign for the close neighbours that dominate in the energy. This is the case for Mo, but fails for $\mathrm{W}$ and $\mathrm{Al}$. Their predictions suggested that for spherical voids one expects $R_{\mathrm{L}}$, the nearest 
neighbour distance for the void lattice, be about three times the void radius. Whilst the lattice is stable, its binding energy is too small in the Malen-Bullough model to explain the stability at high temperatures.

Stoneham (1971) showed that one could give an exact solution in closed form for the energy per void in an array, avoiding some of Malen and Bullough's working assumptions. Voids were represented by defect forces in an anisotropically elastic continuum, with models of all three sources of anisotropy mentioned above. The crucial step is the exploitation of the periodicity of the lattice. A Fourier transform method (as for the shear plane arrays noted above) reduces the expression for the energy per void to a finite sum of terms, without any assumptions of large spacings, weak anisotropy or issues of convergence; moreover, the structure of the underlying host lattice appears naturally. The predicted spacings and energies for Mo are only slightly improved on the values obtained by Malen and Bullough, and, as before, somewhat smaller than observed. Again, approximate proportionality of spacing and radius appears to hold. Stable lattices can be obtained with a variety of defect force configurations having the appropriate symmetry (spherical voids, $\langle 110\rangle$ forces, $\langle 11 l\rangle$ forces but not $\langle 100\rangle$ forces). Since all three contributions to the anisotropy are present (elastic, void shape and host structure) the stability of the lattice does not depend on the sign of $\delta$ alone: stable lattices can occur even in cases of exact elastic isotropy.

Both these models omit two features: phonon dispersion (i.e. the variation of phonon velocity with wave vector; in principle, this could be included in Stoneham's treatment without difficulty), and the effects of the voids on the elastic properties of the host. This second effect, including the induced interaction, was assumed to be small previously because less than $0.10 \%$ of the crystal volume was void. Tewary and Bullough (1972, also Tewary 1973b) developed a model particularly convenient for looking at these two terms. Instead of working with defect forces in a continuum, they divided the crystal into blocks, such that each void corresponded to one missing block. The dynamics of a perfect lattice consisting of the blocks (rather their constituent atoms) was analysed to obtain force-constants from a fit to the phonon dispersion curves. The analysis then used the Fourier transform trick, plus a Green's function method. Tewary and Bullough's main conclusions now agree with experiment. Figure 6 identifies some of the critical components. Specifically, they found a satisfactory spacing to radius ratio. They correctly explained thermal stability (i.e. energy per void to disperse the lattice) as about $0.5 \mathrm{eV}$, and the energy needed to make a void shrink or expand by an amount equivalent to one vacancy per void is about $\mathrm{k}_{\mathrm{B}} \cdot 450^{\circ}$. It is not clear whether phonon dispersion or the induced interactions are more important.

All these theories appear to predict a specific ratio of void size and spacing, leaving the absolute spacing to be estimated by a kinetics-based approach involving the factors which influence void size even in a random distribution.

The current, purely energetic, theories also have problems. First, all published calculations are based on continuum elasticity, albeit with an implicit structure through the Brillouin zone shape. Unpublished atomistic calculations by Finnis $(1980,2006)$ raised doubts by finding only weak void-void interactions. One possible explanation (Stoneham, unpublished 2006) may be that the imperfect symmetry of individual voids and their shape variations might be important. One knows that there are cancellations in the otherwise leading energy terms for spherical voids in isotropic elasticity, so one might expect highly symmetric voids in nearly isotropic systems to have relatively small interactions. We know from the electron micrographs that the voids in the lattice are not very symmetric, and vary randomly in detailed shape from one site to the next. So could the random variations in void shape be leading to interactions enhanced over what one would expect over the highly symmetric models? This factor could underlie the deviations from a strict ratio between spacing and mean diameter. 


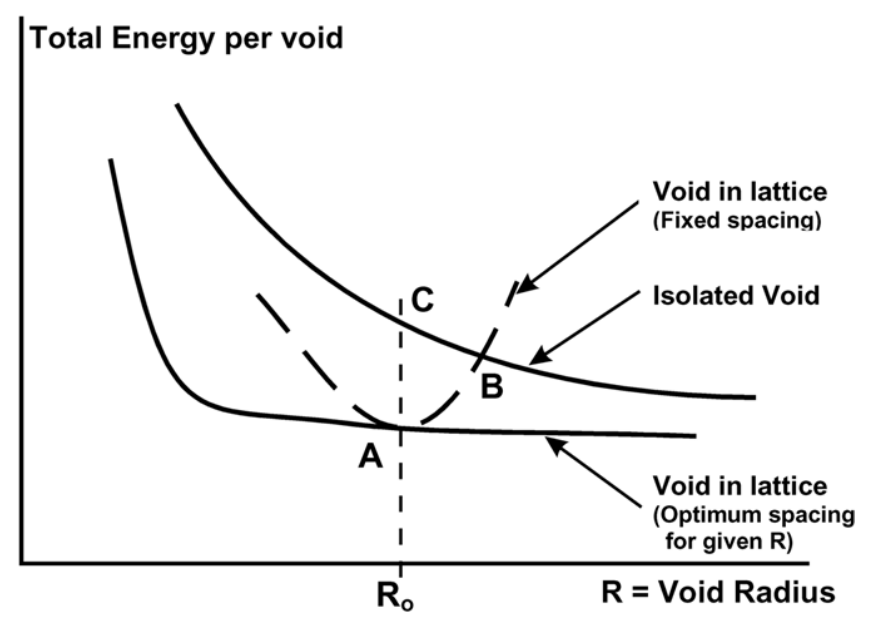

Figure 6. Energies of void systems as a function of void radius, showing stabilization in a void lattice and, once the lattice spacing has been fixed, the extra cost of changing a void dimension. The figure summarizes energetic features: stability against loss of voids, or void lattice melting, stability against growth of all voids at constant lattice spacing. The stability against growth at constant spacing, which leads to saturation, depends on how fast curve II rises above point A, whereas stability against 'melting' is determined by the energy (C-A) at constant void size, or by (B-A) when growth is allowed. Quantitative plots have been obtained in unpublished work of Stoneham and of Tewary.

Emergence of the void lattice. Stoneham (1975) summarized the evidence then available. First, the void lattice only appeared to form when the voids are large enough. Diffraction measurements show it is possible to form high densities of small voids without any signs of long-range order. Secondly, there is no evidence that the voids themselves move in forming the lattice, though his estimates did suggest that surface diffusion was sufficient to let longrange order evolve from pre-existing short-range order. Thirdly, precursors of the void lattice had been reported, e.g. ordering into rows can occur before the three-dimensional structure is apparent $[24,25]$. Fourthly, the void lattice has not yet been formed in metals under electron bombardment, though there are analogies with the ordered colloids observed in $\mathrm{CaF}_{2}(\mathrm{Murr}$ 1974, Chadderton et al 1976).

What happens may start with the formation of many small, randomly distributed voids. As these voids grow and coarsen, local ordered regions are initiated where the void distribution happens fortuitously to be favourable energetically; finally a spread of order to adjacent regions. It is likely that the interaction between the voids is important in this final stage. The kineticsbased theories may provide a more accurate description of earlier stages, and in situations for which void or bubble spacings are short, when the important interactions between growing voids may be less superlattice stabilization, more effects on kinetic parameters. Perhaps the void strain fields influence the emission of vacancies from voids, stabilizing voids lying on the lattice and assisting the evaporation of the others. Work on random voids suggests that a bias effect in emission is more likely than in the capture of vacancies by the voids. However, as noted in the original paper, these hypotheses are tentative, and the role of the detailed void geometry remained to be sorted out. Given the existence of edge dislocations in the superlattice, the role of energy minimization seems essential in the final tidying up of the long-period void lattices. This is not to rule out a modest role for interstitials moving along close-packed directions, but a major role would surely cause elimination of these observed features. 
3.4.6. Miscellaneous ordering mechanisms. There remain miscellaneous forms which are less readily classified.

Vernier and moiré effects, which occur when there is a slight mismatch between two simple periodic systems (e.g. ordinary crystal lattices), have been discussed already (section 2.4). Diffraction effects can lead to structures such as ripples on beaches, these being caused by diffraction of water waves (Bascom 1959).

Surface wave structures. Particles floating on fluids can congregate at specific places on surface waves (Falkovich et al 2005) in ways somewhat analogous to the patterns that Chladni first observed in the 1780s on vibrating plates. Hydrophobic particles drift towards points of maximum vertical displacement; hydrophilic particles tend to cluster at nodes. On a smaller length scale, there can be analogous orderings in aqueous solutions (Howe et al 1974, Neilson et al 1975).

Columnar fracture patterns approximate hexagonal optimal honeycomb patterns. The initial fracture patterns (as seen sometimes in drying mud) have T-like vertices, rather than Y-like. The Y-like ones emerge as thermal strain is reduced, a process that eliminates or modifies poorly positioned junctions (Gray 1986). Gray's mechanism is driven by energetics in a situation that evolves as cooling occurs. One can imagine other mechanisms yielding similar structures, e.g. convection cells in which the solidification process left the walls with a composition of reduced mechanical strength; convection cells are kinetically driven (Chandrasekhar 1961). Such systems do not minimize the free energy, but adopt a structure that gives the maximum initial rate of change of the free energy or similar. Possibly the basalt structures of the Giant's Causeway are of this character. It is sometimes held that bubble and void lattices in irradiated metals arise in this way (Martin 1983, Woo and Frank 1985), and it is likely that this is indeed a component of the story, at least for short-period bubble lattices for which the order is relatively poor.

Particles, Nuts and Pebbles. There is an immense literature on how nuts reorganize when shaken, and just how size and density come in. This is not within the scope of the present survey, except in so far as there can be important influences from short-range order. I would note the finding of Sanders et al (2004) that Brazil nuts (which are large) attract each other in certain regimes when they are among small nuts. The attraction can extend over 4-5 diameters, well beyond the range of common hard-sphere orderings.

\subsection{Key characteristics of periodic organized structures}

Both the analysis by Stoneham (1975) and that of Muthukumar et al (1997) recognize that there are a number of key characteristics of any ordered structure. Whilst the emphasis here is on periodic ordered structures, there will be similar issues for other self-organized systems. These variety of these characteristics leads to a natural set of questions.

What determines the period? Is there a characteristic scale length such that the period is a multiple of that? For convection cells (Chandrasekhar 1961), the characteristic length is the thickness of the convection zone. For shear plane structures (Stoneham and Durham 1973), it is the repeated distance of the crystallographic structure within each of the individual planes that determines the spacings of these planes in an array. For the void lattice, if the elastic interaction dominates, it is the void diameter that determines the lattice parameter of the array (Malen and Bullough 1971). In early studies, there was some controversy, because theory predicted a ratio of lattice parameter to void diameter that was less than observed (see figures 3 and 4). As the experimental database has increased, this seems less of a problem, though 
there is still controversy, and it may be that the short length scale and long length scale lattices involve different mechanisms. The roles of several mechanisms could be complementary, e.g. kinetics determining the average spacings of the voids and initiating short-range order, with diffusion biased by the elastic interaction leading to long-range order. As already noted, it is hard to understand how purely kinetic mechanisms could yield clean dislocations observed in the void lattice.

There can be more than one scale length, and the period is related to them in a way which may be complex. For the fluxoid lattice, there is both a penetration depth and coherence length; in magnetic domain structures there is a demagnetizing energy and a magnetic domain wall energy linked to anisotropy. Wrinkling of a skin, e.g. gelatin on aluminium, shows a lateral periodicity related to the skin thickness. So long as there is a skin that resists short wavelengths, a substrate that resists long wavelengths and certain packing constraints (e.g. clamping), the wavelength of the wrinkles $\lambda$ is related to the skin thickness by $(\lambda / h) \sim$ (elastic modulus of skin/elastic modulus of substrate) $)^{1 / 3}$ (Cerda and Mahadevan 2003, Rizzieri et al 2006).

Does the structure align with the underlying crystal lattice? In some cases-all of class 1 (elasticity ordering) and some of classes 2 (domains in very anisotropic ferromagnets), 4 (long period alloys) and 6 (Vernier structures), the mechanism leading to periodicity automatically ensures alignment. In other cases, e.g. the fluxoid lattice, the alignment observed is the result of small terms which have little or no effect on the ordering (Ullmaier et al 1973). In further cases still (e.g. domains in isotropic ferromagnets) the sample shape may be more important than its underlying structure. Ordering can occur in the absence of a crystal structure (e.g. in liquids).

Can there be defects in the ordered structure? Attractive examples of dislocations, interstitials, etc are seen in the void lattice and the fluxoid lattice. Disclinations are seen in the fluxoid case (Traube and Essmann 1966, 1968) and in domain structures in highly anisotropic ferromagnets (see, e.g. figure 5.5, p 109 of Craike and Tebble 1965). In other cases, whilst the order is not perfect, it is hard to describe it in terms of a 'defect' structure in array; the lack of order is closer to that in amorphous crystals, where short-range order may be maintained with reduced long-range order.

Can one change the ordered structure? The simplest change is to try to alter either the relative sizes of the lattice parameter and the size of a basic unit (e.g. a void diameter) or to try to grow the lattice over a larger region. Various effects are observed (figure 7). One involves growth on the same (pre-existing) sites. For example, domain walls move in response to magnetic field changes. Shear planes grow in non-stoichiometric oxides as the oxygen deficiency spreads across the crystal. However, it seems very rare to see simple growth of voids in a void lattice. In the (disordered) case of the interstitial-H liquid in $\mathrm{Nb}: \mathrm{H}$, the liquid components do seem to grow. A second class of behaviour creates more sites with unaltered components. Examples include the ordered flux lines in type II superconductors as the applied magnetic field changes. Likewise, shear planes may reorganize in this way as the average oxygen deficiency changes. A third variant is saturation: neither growth nor new sites, as for void lattices where extra vacancies usually appear stay in solution once the lattice has formed. 

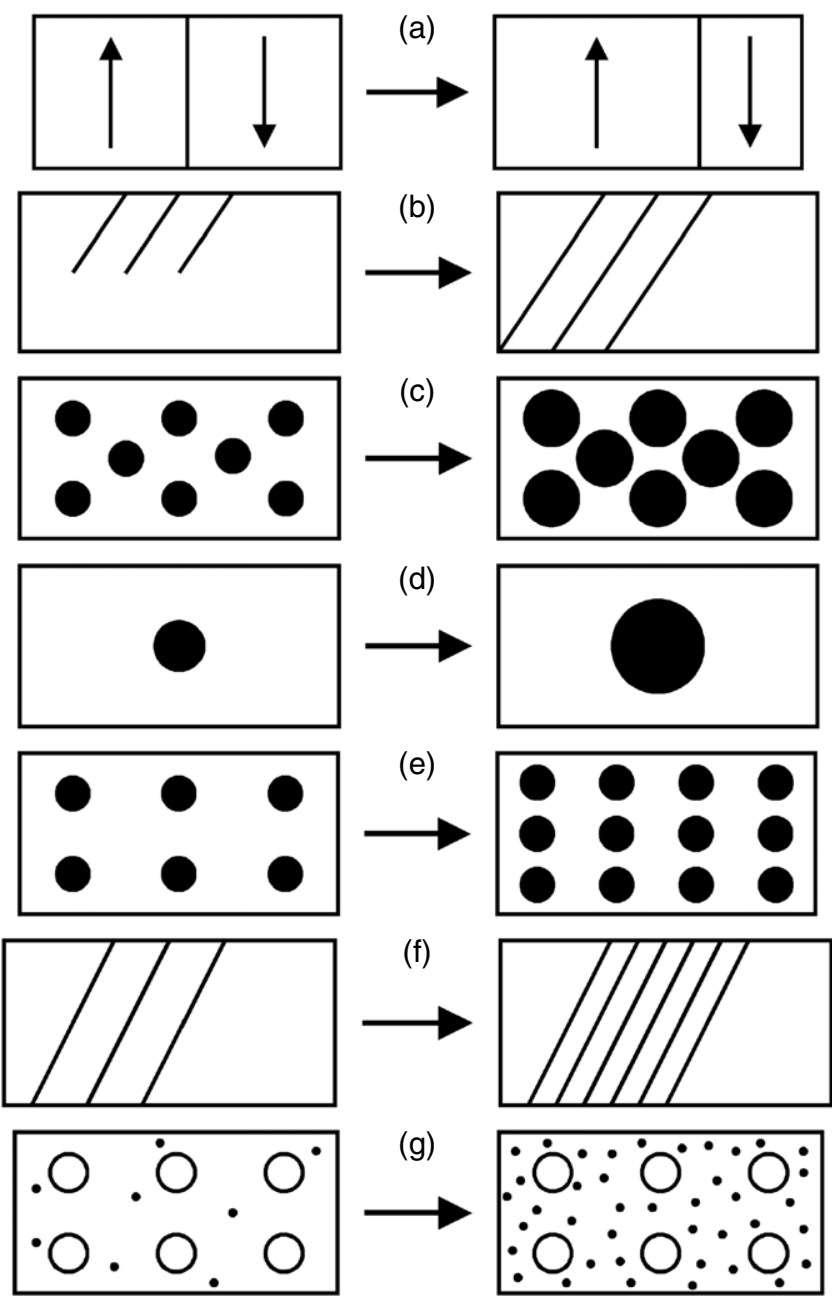

Figure 7. (a) Growth through domain wall motion as an applied field changes. (b) Shear plane extension. (c) This apparently simple case is not normally seen (figure 5 suggests it is costly, at least in some cases). (d) shows an exception to $6(c)$, the disordered interstitial-H liquid in $\mathrm{Nb}: \mathrm{H}$. (e) Some systems change site numbers with components unaltered, such as type II superconductors as the magnetic field changes. $(f)$ Another example: changes in shear planes as the non-stoichiometry increases. $(g$ ) Saturation: no growth or new sites.

\section{Other issues, including robustness, self-organization and chirality}

\subsection{Volume constraints and limits to growth}

The roles of templates. It is striking, and perhaps important, that very few indeed of the many mechanisms in the above classifications of structure and mechanism involve either templating or volume constraints. Meldrum (2003) showed that, in calcite biomineralization, the container or vesicle is important as a mould to produce some complex structures, such as sea-urchin spines. Some of these single orientation networks using moulds apparently need no templating effect at all and no amorphous precursor phase (Park and Meldrum 2004). As Harding (2005) has observed, this implies some remarkable fine tuning on the diffusion versus nucleation times 
because, if the system has one orientation overall, there must be one and only one nucleation event, to avoid differently oriented domains growing in different parts of the mould.

Park and Meldrum show that polymer membranes can act as effective templates for calcite growth. They identify two mechanisms. The first mechanism involves an early calcite nucleation event with subsequent growth; the small (say $40 \mu \mathrm{m}$ ) calcite crystallites show curved surfaces where the crystal has grown in contact with the membrane. There are rhombohedral faces, presumably corresponding to growth fronts into the free space within the membrane and, since such faces are parallel, it would seem there has been a single nucleation event. The subsequent growth and particle sizes depend on conditions such as concentrations of the ingredients in the solutions from which they grow.

The second mechanism involves dissolution of vaterite and precipitation of calcite. Calcite and vaterite may form together, with the less stable vaterite transforming with time. The polymorph proportions can be changed readily; Shao-Feng Chen et al (2006) note that varying the ratios of ethanol and water in solution can give control over the calcite, aragonite and vaterite proportions. Park and Meldrum note some evidence that amorphous calcium carbonate can form thin layers, and that these may recrystallize, but there is no evidence that the templated single crystal calcite must form via such precursors. The ideas of Mann (2001) and Mann et al (1995) suggest $\mathrm{CaCO}_{3}$ forming a gel that coats growing crystals of $\mathrm{CaCO}_{3}$. The later suggestions by Nassif et al (2005) suggest it is some amorphous $\mathrm{CaCO}_{3}$ that results, possibly from an aqueous gel of some sort, but without organics. We know that a relatively soft mould can control the shape of a gel, since this approach underlies some of the tricks of domestic cooking. Provided the amount of material does not grow, shaping makes sense. That being so, a relatively soft (but also relatively impermeable) mould may control the access of material to the growth surface simply by covering it accurately.

Natural size limits. Nanocrystals grown biologically (including inorganic nanocrystals like $\mathrm{CdS}$ and iron oxides) can be constrained to a certain modest size. A suggestion often heard is that the mechanism is 'capping'. But how does the cap constrain the size? One possibility is that it is a matter of optimum fit, like the Caspar and Klug structures (hexamers and pentamers on a nearly spherical surface; see Caspar and Klug (1962), Day (2004), Zandi et al (2004), Twarock (2006)). Another possibility is that the nanocrystal has reached a 'magic number' in size, so there is very little energetic driving force for the next ion/atom to join. However, the variability of nanocrystals (5\% variability in diameter is typical) does not suggest that the magic number effect is strong (even in molecular beam clusters, magic numbers do not dominate).

Size limits that do not invoke limiting the supply of material for growth. If growth occurs on terraces on surfaces, then the soft solid must supply an opposing force related to the thermodynamic energy gain on forming a new layer and to the added thickness per layer, i.e. there could be a structural energy inhibiting growth. It is easier to imagine kinetic limits. Thus, since growth on terraces is often very slow indeed, actual growth may be on a step, or a step kink, or on a screw dislocation (Frank 1952). In at least some of these cases, another molecule may block growth at the special site. Of course, both energetics and kinetics may matter, as for jamming. For example, if there were hard spheres or stiff rods surrounding a growing particle, growth might require significant strain of larger regions (see e.g. Subramanian et al 2005). In a parallel way, oxidation of small silicon spheres or rods is slowed as their sizes reduce. The volume per silicon in the oxide is about twice that in Si itself, and growth occurs at the $\mathrm{Si} /$ oxide interface, so there is a large strain energy opposing growth in smaller particles. 
How a soft solid can act as a mould for a growing hard solid is not easy to understand, especially if there is no obvious restriction on supply of material. Thus ice crystal growth can destroy concrete roads and runways. This may be helped by penetration of moisture to the growth surface, together with debonding in the concrete microstructure. Some stresses come from osmosis and some from stress transmission, as water is almost incompressible. The details will be affected by the fact that confinement does affect melting and freezing, of course (e.g. Alba-Simionescu et al 2006). Free energy gain drives crystal growth, and can be substantial. The control of ice crystal growth plays a role in the texture of ice cream, where the Ostwald ripening of ice crystals affects the 'mouthfeel'. Such recrystallization has parallels in the possible intracellular ice growth that has forced fish, plants and insects to create antifreeze proteins (Koop 2006). It appears that there is a dynamic equilibrium between the ice and the protein on the ice surface (Li et al 2006). When conditions (protein concentration, binding energy, number of binding sites) are appropriate, the protein can inhibit growth down to a predictable degree of cooling below the usual freezing temperature.

More puzzling is the fact that a growing mushroom can crack concrete, which admittedly is weak under tension. The growing mushroom can exert a pressure of just under an atmosphere (tens of $\mathrm{kPa}$, Buller 1920), a mere $10^{-5} \mathrm{eV}$ per surface site, a pressure that could not block crystal growth. Yet the relatively soft solid is not constrained effectively by the hard one.

Mechanisms involving limits on supply of material. The more probable situations might involve diffusion barriers, or perhaps some threshold or concentration-related problem even if there has been successful nucleation. In such cases, there could be a role for additives, with the other common control parameters $(T, p$, reactant flow, etc) having possible influence. Electrostatics, or imbalance of reactants can combine in various ways. One possible limit involves diversion of reactants through competition between different nucleation sites.

One particular example in which supply is limited involves protein cages, these being crucial in the synthesis of magnetic nanoparticles. Such particles, often hydrated ferric oxides or phosphates (see e.g. Klem et al 2005), include the single domain ferrimagnetic $\mathrm{Fe}_{3} \mathrm{O}_{4}$ found in magnetotactic bacteria. Examples of such cages in order of increasing internal diameter include tobacco mosaic virus (internal diameter $4 \mathrm{~nm}$, external diameter $18 \mathrm{~nm}$ ), horse spleen ferritin (internal $8 \mathrm{~nm}$, external $12 \mathrm{~nm}$ ) and cowpea chlorotic mottle virus (internal $24 \mathrm{~nm}$, external $28 \mathrm{~nm}$ ). The mammalian ferritins are built from two types of subunit $(\mathrm{H}, \mathrm{L})$, both comprising a four-helix bundle capped by a helical domain lying at about $60^{\circ}$ to the bundle axis. The subunits align in 12 sets of antiparallel pairs, giving a roughly rhombic dodecahedron shape. The packing forms a shell, with $\sim 3 \AA$ diameter channels. Those channels at threefold axes are mainly hydrophilic, whereas those at fourfold axes (surrounded by four helices from different subunits) are largely hydrophobic. The mineralization process includes oxidation of $\mathrm{Fe}(\mathrm{II})$, hydrolysis, nucleation, and growth. The $\mathrm{Fe}(\mathrm{II})$ ions enter the cage via the hydrophilic threefold channels. Electrostatics is important: the outer entrance is a region of positive potential, giving a field that guides the cations into the cage. In the case of ferritin, magnetic measurements suggest the iron oxide produced (either $\gamma-\mathrm{Fe}_{2} \mathrm{O}_{3}$ or $\mathrm{Fe}_{3} \mathrm{O}_{4}$ ) fills the internal cavity rather precisely, and incorporates a few thousands of $\mathrm{Fe}$ ions. Presumably electrostatic repulsion is the underlying physical mechanism that stops growth. It would seem probable that similar mechanisms are involved when yeasts produce CdS nanocrystals (Williams et al 1996), and also how bird's brains keep magnetite crystals small. There are possible analogies here with conventional inorganic systems, such as the growth of InP nanoparticles into zeolites (Agger et al 1998). 
Clever control of size. This category would include the ways that living organisms exploit the capabilities of DNA. Many of the processes that controls cell sizes can be rationalized in terms of the combination of standard physical processes, like diffusion, and the special capabilities of DNA, RNA and proteins. But multicell organisms must also control both overall size and organ size (see e.g. Bjorklund et al 2006). When one turns to large complex structures, like the elegantly spiral shells of Nautilus pompilius (D'Arcy Thompson 1917, p 173), more than one mechanism may be involved. How does 'clever' biocontrol (encoded in DNA) operate for complex structures like shells? There are several possibilities. One is that the DNA determines the structure of the mould that the biomineral must fill. But the crystalline biomineral must be prevented from growing through the mould. This might be achieved by judicious blocking of growth sites, or possibly be ingenious cutting down of the growth materials. Blocking would be easiest if there is only a need to block specific sites, e.g. if growth is on a step, if growth is on a screw dislocation, or if growth occurs at specific kink sites. It seems unlikely that supply of material suffices for control, and it is not altogether clear how protein inhibition at an interface would be controlled to give the rather precise overall structure observed. It is not a full answer to say that there is a soft mould, since that mould must itself be shaped. Understanding the way in which these fascinating structures are controlled would presumably open some important opportunities for self-organized technological structures.

\subsection{Selectivity in the presence of fluctuations}

Templating suggests that two surfaces, a substrate and a growing film, have shared structural features, i.e. that one structure grows instead of alternative forms. Receptors also share structural features with some molecules rather than others, such that only a very small number of molecular species may be able to actuate the receptor. For large molecules, the actuation is probably mechanical or electrostatic (e.g. Honig and Nicholls 1995), i.e. the molecule fits fairly well into the receptor, but there are forces that then open a channel or similar. For small molecules, it is clear that this 'lock and key' description is insufficient. In olfaction, for instance, molecules of essentially identical shape smell wholly different (Turin 1996). There is some additional feature, perhaps an electron transfer or a proton transfer, that actuates the receptor, which is thus more like a swipe card reader than a lock (Brookes et al 2007). In such cases, there are two sources of selectivity. The molecular shape must match the receptor well enough, and the molecules must be able to have the extra feature for the actuation step. The problems of shape-alone interpretations are exacerbated by thermal fluctuations that, in living things, are so significant that nature does not seem to attempt to achieve perfect fidelity using repair enzymes (Radman 2001), a situation far removed from average solid-state modelling.

These ideas may apply in other situations. For example, the free energy differences favouring one growth mode over another are often really rather small. Given that there are many mechanisms giving mesoscopic order that are not. based on templating or volume constraints, the question is this: is there only one factor that pushes a system into a particular orientation or structure? One might link this question to Hopfield's (1974) discussion of kinetic proofreading, in which a two-step route is needed to ensure that the right metabolic reaction occurs in cases where the differences in activation energy do not discriminate enough in a single step.

\subsection{Robustness}

The robustness of an organized structure relates to the accuracy of the final structure and to the range of conditions over which that structure is formed. Examples have already been given 
for void lattices, domain structures and periodic systems. For organisms, the most important robustness issue concerns function: does the organized structure work adequately?

The robustness of a structure might mean the accuracy in degree of alignment or of epitaxy with a substrate, or the extent to which defects are allowed and actually happen. But other factors are important. How easily can the structure be changed (domain wall shift, shear plane extension, more sites added on, etc)? There are standard approaches to address these questions for systems that are in equilibrium, and also for some simple near equilibrium systems. Nevertheless, there remain unexpected examples of relatively robust behaviour, such as graphoepitaxy and the memory of crystal orientation through an amorphous phase.

Robustness applies to processes as well as to structures. Again, there are cases which seem robust yet it is not clear why. An example might be sonocrystallization, in which an intense sonar beam eliminates some undesired crystalline forms, presumably through non-equilibrium processes. Perhaps this is another reminder of how much more remains to be known about nucleation. The nucleation step can be especially sensitive to details, i.e. not robust. For instance, the stabilization of voids over the lower energy dislocation loops appears to need a small number of radiation-produced alpha particles.

\subsection{Chirality}

Left- and right-handed versions should be degenerate in energy. Yet frequently one finds a predominance of just one of these forms. Thus chirality is even found in inorganic systems, e.g. $1 \%$ excess of L-quartz over D-quartz worldwide. The reason is not clear; it seems too substantial an effect to come from parity non-conservation, but may reflect the Earth's rotation and disposition of the land mass.

An important observation (Soai et al 1995) is that homochiral formation of an organic molecule could be achieved with the help of chiral inorganic crystals (in this case, sodium chlorate, as also used by Kondepudi). As Soai noted, inorganic crystals were around when life on Earth began. One can thus see a sequence of possible events that gave the molecules of life their unique chiralities. When calcite grows in the presence of either L- or R-aspartic acid, an asymmetric morphology of steps and terraces emerges (Orme et al 2001, Addadi and Weiner 2001).

In self-catalysis, e.g. in a system away from equilibrium, a slight imbalance, can lead to homochirality. This idea has parallels with Belouzov-Zhabotinsky oscillatory chemical reactions (see Noyes and Field 1974) in which autocatalysis is a key factor, but the symmetry broken does not relate to chirality. It is certainly true that chirality can propagate from small (molecular scale) structures to the macromolecular level. Thus Blüm et al (2005) have shown that the chiral molecule rubrene $\left(\mathrm{C}_{42} \mathrm{H}_{28}\right)$, a small buckled sheet of benzene rings, organizes on a gold surface to produce a wealth of homochiral structures: first pentagonal rings, then chains or ten-membered rings.

Homochirality can be induced by stirring a saturated solution as crystals begin to form (Kondepudi et al 1990). What seems to be happening is the primary nucleation of (say) the L form, the suppression of further primary nucleation but the initiation of secondary nucleation on the first nucleus (with the same chirality as their substrate), and then the breaking off of the secondary nuclei to start separate crystals. This idea was extended and modelled by Cartwright et al (2004). A related experiment (Viedma 2005) used grinding: the crystals just formed were crushed between glass marbles. Yet again, one chiral form emerges, apparently partly through Ostwald ripening to reinforce a particular chiral balance. As in section 4.2, one notes a two step selection mechanism. One should not be too hasty to assume a single nucleation event is the whole story. 
Vainstein (1986) makes the point that biological macromolecules are built from asymmetric small molecules, and that chiral features are pervasive. His discussion of the symmetry aspects of biological macromolecular structures links to the views of Herrmann (1986), who has noted that basic cell functions are associated with asymmetric structures. Specific examples include the way that extracellular molecular signals are transduced into intracellular responses by the cell surface membrane, the syntheses of messenger RNA and of cell proteins and the mechanism of the conversion of metabolic energy into mechanical work, as in muscle contraction. Whether chirality is necessary in all such cases is unclear. Nor is it clear that molecular chirality links directly to mesoscale and macroscopic chirality of organisms, though this seems likely. Ball (2004, p 146) cites studies of growth patterns of bacillus cells that show chiral patterns of several sorts, such as curling tendrils or spiralling arms ending in blobs (Ben-Jacob 1993, Ben-Jacob et al 1994, 1995, 1998, Fujikawa and Matsushita 1989). These chiral patterns imply coordinated motions, rather than the simpler quasi-fractal forms of diffusion-limited aggregation seen in electrodeposition and analogous cases (Witten and Sander 1981, Ball et al 1984). Somewhat similar issues enable accurate cell division in E. coli (Kulkarni et al 2004) through the diffusion controlled polar localisation of proteins.

Some unexpected formal results are indicated by Sokolov (1986) in a discussion of chirality in stereochemistry. First, he observes that Noether's theorem means there is an invariant 'incidental to the continuous zone of chiral conformations, that has an achiral boundary'. The types of structures for which such zones exist are determined by another theorem, due to Ruch. However, the configuration cannot be determined by purely chemical means; this, Sokolov says, follows from Gödel's theorem. The interesting point here is that there would seem to be good and rather fundamental reasons for expecting chirality to emerge, even if the precise mechanisms are unclear.

\section{Conclusions: bringing organization to self-organization}

Self-organization is a poorly defined term, encompassing phenomena from the simple sorting of shaken nuts by size to the highly complex life processes controlled by DNA. Within this range are some closely defined approaches that lead to order, whether at the atomic scale, at the mesoscale or at the macroscopic scale. This review has tried to give some systematic order to the immense variety of organizing phenomena. My emphasis has been on how organization can be achieved by physical mechanisms not involving DNA. Of course, even DNA operates in a physical world, but I have not attempted to discuss the way that information encoded in DNA becomes operational through control of the forces and the energies that determine the structures and performances of organisms.

I have made no attempt to claim that there is one single underlying principle, for clearly there is no such principle. Nor would one wish to claim self-organization is rigidly precise, either in a molecular sense or in an engineering sense. Indeed, exactness must surely be compromised when there are both ideas implying some fixed length scale for living things and yet also widely discussed biological scaling laws.

Mechanisms for mesoscale order seem surprisingly common. There are inevitably various possible outcomes, and competition between the several control factors. The major routes to mesoscale order fall into five major categories. Clearly, free energy minimization is one possibility. Then there is control through dynamics on an energy surface near equilibrium (diffusion; precipitation; nucleation). Thirdly, the topology of the energy surface near equilibrium may be critical, when configurational entropy is more important than enthalpy. Geometrical guidance (templates, chiral nuclei) can take many forms, including also the sorting of balls of various sizes. Finally, the outcome may be selected as a fastest-forming 
instability, possibly involving a sequence of structures with branching points in their evolution (Thom 1983).

Given this variety, is there a way to reach a tightly defined final state starting from a relatively featureless initial state? Can this be achieved without clever DNA tricks or, if not, can one identify how DNA achieves these tricks? At the mesoscale, a number of questions emerge. Can one pre-select a length scale, other than by templating? What happens quite often is that there are simple relations between two characteristic lengths (see, e.g. section 3.4.1). How stable is stable enough? How big a difference in critical energies suffices? How does nature manage with only small energy differences and narrow temperature ranges? Is it by using a proofreading trick? To what extent can phase diagrams describe the various behaviours? Muthukumar et al (1997) give an example of competing interactions and sequences, devising a chain with four types of domain, each with some functionality, where different sequences of domains lead to different degrees of stability. Prior structures (the first-formed ones) impose constraints on the subsequent structures, e.g. this is why voids exist when they are massively more energetic than dislocation loops. So at which stage is an observed structure determined? Nucleation is not just a matter of size. Muthukumar et al (1997) note synergistic interactions can be a key element, when the first interaction to create order gives important constraints on behaviour at other length scales. They discuss cases including $\mathrm{H}$-bonds and $\pi$-stacking bonds. How do living organisms balance natural length scales (those that are determined by clear physical principles; see Stoneham and Harding (2003)) and operational length scales (those that optimize performance)? A similar balance is needed between natural and operational timescales. Nature seems to manage self-organization in ways we cannot always achieve in synthetics. These include spontaneous selection of primary length scales (and symmetries), especially competing interactions, and how scale lengths might be tuned. Achieving order at the atomic scale seems possible with ingenious templating, but it is non-trivial to go beyond this to produce some hierarchy of larger structures, especially if there should be controlled order at the longer scales. But perhaps our scientific intuition focuses too much on precision (i.e. accurate order parameters) rather than accuracy of key elements. Biomolecules like DNA get right those features that must be right, allowing considerable freedom to features that are inessential.

There remain important unsolved questions at other scales. At the atomic scale, nucleation remains problematic. We all know the simple descriptions that relate nucleation barriers to surface and bulk energies, but these models are rarely realistic, either in geometric detail or in suggesting how a particular phase or orientation is chosen, especially in the presence of fluctuations that might render a template inaccurate. The selectivity issue is a difficult one when there is only a modest energy difference between alternatives, so that a simple Boltzmann factor is not very selective. The difficulties increase when there is a complex energy landscape in which there are varied competing free energy minima (e.g. Wales 2003, Brooks et al 2001). In some systems, one sees high energy structures (like voids in irradiated metals) emerging, even though dislocation loops would be far more stable. In that case, the presence of voids appears to be due to small amounts of fission gas that has a critical effect on small growing vacancy aggregates, a decisive factor that is very easily ignored. A second unresolved question is how much of organization at the mesoscale relies on templates, since there seems to be a wide diversity of mechanisms that do not need templates? And how many mesoscale structures exploit more than one organizational technique, e.g. one to fix a scale and the other to select a geometry? Thirdly, at the macroscopic scale, how can one stop the growth of an inorganic material in a precise way, as in making a shell? Whereas, for example, finger nails and hair grow only to certain lengths, they do so without precision; shells can have complex architectures over significant differences, so that some control seems implicit on the 
scale of the shell dimensions. There are many suggestions as to how mesoscale structures are organized, including interesting hierarchical strategies, but it is less clear how growth is organized at, say, the $0.1 \mathrm{~m}$ scale. This issue of how growth is stopped is, however, just one example of even broader issues of bio-organization, and especially in understanding just how DNA ultimately operates through physical mechanisms.

\section{Acknowledgments}

This work was supported in part by EPSRC through its Materials Modelling Initiative and Basic Technologies programmes. Some of the earlier work was supported by the Underlying Research programmes of UKAEA. The author is greatly indebted to many friends and colleagues for input and discussions, notably John Harding and Dorothy Duffy, and to John Evans for providing the superb micrograph of the void lattice.

\section{References}

Ackerson B J and Clark N A 1984 Phys. Rev. 30 906-18

Adair J H and Suvaci E 2000 Curr. Opin. Colloid Interf. Sci. 5160

Addadi L and Weiner S 1997 Nature 389 912-15

Addadi L and Weiner S 2001 Nature 411753

Agger J R, Anderson M W, Pemble M E, Terasaki O and Nozue Y 1998 J. Phys. Chem. B 1023345

Aizenberg J, Black A J and Whitesides G M 1999a Nature 398495

Aizenberg J, Black A J and Whitesides G M 1999b J. Am. Chem. Soc. 1214500

Aizenberg J, Tkachenko A, Weiner S, Addadi L and Hendler G 2001 Nature 412819

Alba-Simionescu C, Coasne B, Dosseh G, Dudziak G, Gubbins K E, Radhakrishnan R and Sliwinska-Bartkowiak M 2006 J. Phys.: Condens. Matter 18 R 15

Alerhand O L, Vanderbilt D, Meade R D and Joannopoulos J D 1988 Phys. Rev. Lett. 61 1973-6

Anderson J S 1972 Reactivity of Solids (London: Chapman and Hall) p 1

Antonietti M and Ozin G A 2004 Chem. Eur. J. 1028

Aranda-Espinoza H, Chen Yi, Dan N, Lubensky T C, Nelson P, Ramos L and Weitz D A 1999 Science 285394

Ardell A J and Nicholson R B 1966 Acta Metall. 141295 (with an appendix by J D Eshelby starting on p 1306)

Autumn K et al 2002 Proc. Natl Acad. Sci. USA 9912252

Ball P 2004 Critical Mass: How One Thing Leads to Another (Portsmarth, NH: Heinemann) (s.1.) p 146

Ball R, Nauenberg M and Witten T A 1984 Phys. Rev. 292017

Ball C A B and van der Merwe J H 1983 Dislocations in Solids ed F R N Nabarro (Amsterdam: North-Holland) p 122

Banga S, Chawla G and Bansal A K 2004 Pharmagenerics Business Briefing p 70 http://www.touchbriefings.com/pdf/ 955/ACFB38E.pdf

Barthlott W and Neinhuis C 1997 Planta 2021

Bascom W 1959 Sci. Am. 20041

Bejan A 2000 Shape and Structure: From Engineering to Nature (Cambridge: Cambridge University Press)

Ben-Jacob E 1993 Contemp. Phys. 34 247-73

Ben-Jacob E, Cohen I and Gutnick D L 1998 Annu. Rev. Microbiol. 52 779-806

Ben-Jacob E, Sochet O, Tenenbaum A, Cohen I, Czirok A and Vicsek T 1994 Nature 368 46-9

Ben-Jacob E, Sochet O, Tenenbaum A, Cohen I, Czirok A and Vicsek T 1995 Phys. Rev. Lett. 752899

Bennema P 1991 Sir Charles Frank OBE FRS: An 80th Birthday Tribute ed R G Chambers et al (Bristol: Adam Hilger) p 46

Berman A and Charych D 1999 J. Cryst. Growth 198-199 796-801

Bernal J D and Fankuchen I 1941 J. Gen. Physiol. 25111

Björklund M et al 2006 Nature 4391009

Blüm M-C, Ćavar E, Pivetta M, Patthey F and Schneider W-D 2005 Angew. Chem. Int. Edn 44 5334-7

Bradley D 1995 New Sci. p 18

Braterman P S and Cairns-Smith A G 1987 Orig. Life 17221

Bravais A 1913 Etudes Crystallographiques (Paris: Academie des Sciences)

Brookes J, Hartsiou F, Horsfield A and Stoneham A M 2007 Phys. Rev. Lett. 98038101 
Brooks C L, Onuchic J N and Wales D 2001 Science 293612

Brune H, Giovannini M, Bromann K and Kern K 1988 Nature 394451

Buller R 1998 The Last Word (Oxford: Oxford Paperbacks) p 24

Bullough R, Eyre B L and Krishan K 1975 Proc. R. Soc. A 34681

Burgess D S 2005 Photonics Spectra p 121

Burton W K, Cabrera N, Frank F C 1951 Phil. Trans. R. Soc. A 243299

Cahn R W 1995 Nature 375363

Cahn J W 1961 Acta Metall. 9795

Cahn J W 1968a Trans Metall. Soc. AIME 242166

Cahn J W 1968b J. Appl. Phys. 394052

Cahn J W, Taylor J E and Handwerker C A 1991 Sir Charles Frank: An Eightieth Birthday Tribute ed R G Chambers et al (Bristol: Adam Hilger) p 88

Cairns-Smith A G 1982 Genetic Takeover and the Mineral Origin of Life (Cambridge: Cambridge University Press)

Campbell A L et al 1999 J. Struct. Biol. 126105

Cao Z et al 2005 Science $\mathbf{3 0 9} 909$

Capellini G, De Seta M, Evangelisti F, Zinovyev V A, Vastola G, Montalenti F and Miglio L 2006 Phys. Rev. Lett. 96106102

Carlsson A E and Meschter P J 1989 J. Mater. Res. 4 1060-3

Cartwright J H E et al 2004 Phys. Rev. Lett. 93035502

Casper D L D and Klug A 1962 Cold Spring Harbor Symp. Quant. Biol. 271

Cassie A D B and Baxter S 1944 Trans. Faraday Soc. 40546

Catlow C R A 1979 Int. Conf. on Modulated Structures (Hawaii, March 1979) also Non-stoichiometric Oxides (New York: Academic) p 61

Cavalli S, Popescu D C, Tellers E, Overhand M, Rapaport H, Sommerdijk N A J M and Kros A 2006a Polym. Mater 94577

Cavalli S, Popescu D C, Tellers E, Vos M R J, Pichon B, Overhand M, Rapaport H, Sommerdijk N A J M and Kros A 2006b Angew. Chem. Int. Edn 118 753-8

Cerda E and Mahadevan L 2003 Phys. Rev. Lett. 9074302

Chambliss D D, Wilson R J and Chiang S 1991 Phys. Rev. Lett. 661721

Chadderton L T, Johnson E and Wohlenberg T 1976 Comment. Solid State Phys. 7105 also Monograph 76-12, Physics Laboratory, H C Oersted Institute, Copenhagen University

Chandrasekhar S 1961 Hydrodynamics and Hydromagnetic Stability (Oxford: Oxford University Press)

Chen S-F, Yu S-H, Jiang J, Li F and Liu Y 2006 Chem. Mater. 18115

Cheng Y-T and Rodak D E 2005 Appl. Phys. Lett. 86144101

Clarkson E N K and Levi-Setti R 1975 Nature 254 663-7

Cook H E and de Fontaine D 1971 Acta Metall. 19607

Cormack A N, Tasker P W and Catlow C R A 1982 UKAEA Report AERE TP 963

Craike D J and Tebble R S 1965 Ferromagnetism and Ferromagnetic Domains (Amsterdam: North-Holland)

Croset B, Girard Y, Prevot G, Sotto M, Garreau Y, Pinchaux R and Sauvage-Simkin M 2002 Phys. Rev. Lett. 88056103

Cuvelier D and Nassoy P 2004 Phys. Rev. Lett. 93228101

Day C 2004 Phys. Today 29

Dee G T 1986 Phys. Rev. Lett. 57275

de Fontaine D 1973 Phase Transitions and Their Applications in Materials Science, University Park, PA, 1973 ed L E Cross (New York: Pergamon) p 169

de Fontaine D and Cook H E 1970 Critical Phenomena in Alloys, Magnets and Superconductors, Geneva and Gstaad, Switzerland, 1970 (New York: McGraw-Hill) p 257

de Gennes P G 1986 Directions in Condensed Matter Physics ed G Grinstein and G Mazenko (Singapore: World Scientific) p 83

Delbruck M 1962 Mathematical problems in the biological sciences Proc. Symp. Appl. Math. 1455

Dennehy R D 2003 Org. Process Res. Dev. 71002

den Wolde P R and Frenkel D 1997 Science 2771975

De Rosier D J et al 1980 Nature 287291

Doan N V and Martin G 2003 Phys. Rev. B 67134107

Donnay J D H and Harker D 1937 Am. Mineral. 22463

Duffy D M and Harding J H 2002 J. Mater. Chem. 123419

Duffy D M and Harding J H 2004a Langmuir 207630

Duffy D M and Harding J H 2004b Langmuir 207637 
Duffy D M and Harding J H 2005a Langmuir 213850

Duffy D M and Harding J H 2005b Surf. Sci. 595151

Duffy D M, Travaille A M, van Kempen H and Harding J H 2005 J. Phys. Chem. B 1095713

Efremov I F 1976 Surf. Colloid Sci. 8 85-192

Evans J H 1971 Nature 229403

Evans J H 1971 Radiat. Eff. 1055

Evans J H 1990 NATO Workshop on Patterns, Defects and Materials Instabilities ed D Walgraef and N M Ghoniam (Dordrecht: Kluwer) p 347

Evans J H 2006 Phil. Mag. 86173

Evans J H, Bullough R and Stoneham A M 1972 US Atomic Energy Commission Symp. Series 26 ed J W Corbett and L C Ianiello p 522

Eyre B L and Bartlett A F 1973 J. Nucl. Nat. 47143

Falkovich G, Weinberg A, Denissenko P and Lukaschuk S 2005 Nature 4351045

Finch A I and Quarrell A G 1934 Proc. Phys. Soc. 48148

Finney J, Daniel R and Stoneham A M (issue ed) 2004 Phil. Trans. R. Soc. B 3591141

Finnis M W 1980 unpublished calculations

Finnis M W 2006 private communication

Foreman A J E 1972 UKAEA Harwell Report AERE R-7135

Forty R 2001 Trilobite! Eyewitness to Evolution (London: Flamingo)

Frank F C 1952 Adv. Phys. 191

Frank F C 1958 Growth and Perfection of Crystals ed R H Doremus et al (New York: Wiley) p 411

Frank F C 1972 Z. Phys. Chem. NF 7784

Frank F C 1983 Phys. Bull. (letter)

Frank F C and van der Merwe J H 1949a Proc. R. Soc. A 198205

Frank F C and van der Merwe J H 1949b Proc. R. Soc. A 200125

Freeman C L, Claeyssens F, Allan N L and Harding J H 2006 Phys. Rev. Lett. 96066102

Frenkel D 2006 Nature 443641

Frenkel J and Kontorova T 1938 Phys. Zeit Sowjet 1311

Friedel G 1907 Bull. Soc. Fr. Mineral. Crystallogr. 30326

Frisch H L and Wasserman E J 1961 J. Am. Chem. Soc. 833789

Fujikawa H and Matsushita M 1989 J. Phys. Soc. Japan 58 3875-8

Geiger H 1986 see Hargittai p 329

Gengel L 1978 Phys. Lett. A 65371

Ghoniem N M, Walgraef D and Zinkle S J 2002 J. Comput. Aided Des. 81

Gosselin L and Bejan A 2005 J. Appl. Phys. 98104903

Gould S J 1993 Eight Little Piggies (London: Jonathan Cape) p 453

Gould S J 1991 Introduction to D'Arcy Thompson (Cambridge: Cambridge University Press)

Gray N H 1986 see Hargittai 1986 p 531

Guy A G 1972 Introduction to Materials Science (New York: McGraw-Hill) esp pp 318-47

Haldane F D M and Villain J 1981 Commensurate-incommensurate transitions of physisorbed films on anisotropic substrates J. Physique 42 1673-90

Hämäläinen M 1967 J. Cryst. Growth 1125

Hämäläinen M 1968 J. Cryst. Growth 2131

Harding J H 2005 private communication

Harding J H and Duffy D M 2006 J. Mater. Chem. 161105

Hargittai I 1986 Symmetry: Unifying Human Understanding (Oxford: Pergamon) also issued as Comput. Math. Appl. B 121

Hawthorne F C 1985 Am. Mineral. 70455

Hayes W and Stoneham A M 1985 Defects and Defect Processes in Non-metallic Solids (New York: Wiley)

Hedges E S 1932 Liesegang Rings and other Periodic Structures (London: Chapman and Hall)

Herrmann C H 1986 Hargittai p 155

Heywood B R and Mann S 1994 Chem. Mater. 6 311-18

Hillert M 1965 Acta Metall. 9525

Honig B and Nicholls A 1995 Science 2681144

Hopfield J J 1974 Proc. Natl Acad. Sci. USA 71 4135-9

Howard J 2001 Mechanics of Motor Proteins and the Cytoskeleton (Sunderland, MA: Sinauer Associates)

Howe R A, Howells W S and Enderby J E 1974 J. Physique C 7 L111

Iguchi E and Tilley R J D 1977 Phil. Trans. R. Soc. 28655 
Itoh N and Stoneham A M 2001 Materials Modification by Electronic Excitation (Cambridge: Cambridge University Press)

Jäger W, Ehrhart P and Schilling W 1988 Solid State. Phenom. 3-4 297

Jäger W, Ehrhart P, Schilling W, Dworschak F, Gadalla A A and Tsukuda N 1987 Mater. Sci. Forum 15-18 881

Jain S C, Gosling T J, Willis J R, Totterdell D H J and Bullough R 1992 Phil. Mag. A 651151

Johnson E 1977 Scand. J. Metall. 631

Johnson P B and Mazey D J 1979 Nature 281359

Johnson P B, Thomson R W and Mazey D J 1990 Nature 347265

Jones R A L 2004 Soft Machines (Oxford: Oxford University Press)

Kahlweit M 1965 Prog. Solid State Chem. 2134

Kai S et al 1982 J. Chem. Phys. 761392

Kasper E and Herzog H J 1977 Thin Solid Films 44357

Kasper E, Herzog H J and Kibbel H 1975 Appl. Phys. 8199

Khachaturyan A G 1968 Sov. Phys. Solid State 92249

Khachaturyan A G 1978 Prog. Mater. Sci. 221

Kiely C J et al 1998 Nature 396444

Kirby A J 1993 Phil. Trans. R. Soc. 34567

Kirkaldy J S 1992 Rep. Prog. Phys. 55723

Kitahata H and Yoshikawa K 2005 J. Phys.: Condens. Matter 17 S4239

Kittel C 1996 Introduction to Solid State Physics 7th edn (New York: Wiley) p 301

Klem M T, Young M and Douglas T 2005 Mater. Today p 28

Klue R L and Mullins W W 1969 Acta Metall. 1759

Klue R L and Mullins W W 1969 Acta Metall. 1769

Koch A J and Meinhardt H 1994 Rev. Mod. Phys. 661481

Kohn R V and Müller S 1994 Commun. Pure Appl. Math. 47 405-35

Kondepudi D K, Kaufman R J and Singh N 1990 Science 250975

Koop T 2006 Nature $\mathbf{4 4 2} 333$

Krishnamoorthy S, Hinderling C and Heinzelmann H 2006 Mater. Today 940

Krishan K 1982a Radiat. Eff. 66121

Krishan K 1982b Phil. Mag. A 45401

Krishan K 1988 Solid State Phenom. 3-4 267

Kulkarni R V, Huang K C, Kloster M and Wingreen N S 2004 Phys. Rev. Lett. 93228103

Kumar S, Jae-Hoon Kim and Yushan Shi 2005 Phys. Rev. Lett. 94077803

Küther J, Seshadri R, Knoll W and Tremel W 1998 J. Mater. Chem. 8641

Lehmann A, König G, Rieder K-H 1994 Phys. Rev. Lett. 733125

Leubner I H 2000 Curr. Opin. Colloid Interface Sci. 5151

Li et al 2006 J. Chem. Phys. 124204702

Liesegang R E 1896 Naturewirs Wochschr. 11353

Liu Y, Guo Y, Valles J M and Tang J X 2006 Proc. Natl Acad. Sci. 19310654

Littlewood P B and Heine V 1981 J. Phys. C: Solid State Phys. 142943

Longo R C, Stepanyuk V S and Kirschner J 2006 J. Phys.: Condens. Matter 189143

Mackay A L 1986 Symmetry: Unifying Human Understanding (Oxford: Pergamon) p 21

Magnussen O M, Ocko B M, Deutsch M, Regan M J, Pershan P S, Abernathy D, Grübel G and Legrand J-F 1996 Nature 384250

Malen K and Bullough R 1971 Proc. Brit. Nucl. Eng. Soc. Reading Conf. (Reading, UK) ed S F Pugh et al p 109

Mann S 2001 Biomineralization, Principles and Concepts in Bioinorganic Materials Chemistry (Oxford: Oxford University Press)

Mann S, Didymus J and Sims S 1995 Chem. Commun. 1031

Mann S, Heywood B R, Rajam S and Walker J B A 1991 J. Phys. D.: Appl. Phys. 24 154-64

Marchenko V I and Parshin A Y 1981 Sov. Phys._JETP 52129

Martin G 1983 Phys. Rev. Lett. 50250

Matthews J W and Blakeslee A E 1977 J. Vac. Sci. Technol. 14989

Matthews R A J 1995 Eur. J. Phys. 16172

Mauroy B, Filoche M, Weibel E R and Sapoval B 2004 Nature 427633

Meinhardt H 1992 Rep. Prog. Phys. 55797

Meldrum F 2003 Int. Mater. Rev. 48187

Milchev A and Markov I 1984 Surf. Sci. 136503

Milchev A and Markov I 1984 Surf. Sci. 145313 
Millman B M, Irving T C, Nickel B G and Loosely-Millman M E 1984 Biophys. J. 45551

Moteff J, Sikka V K and Jang H 1974 Harwell Voids Symp. (Harwell, UK) ed R S Nelson p 181 (AERE R-7934)

Müller S 2007 http://www.mis.mpg.de/sm/microstr/microstr-jahrbuch.html

Mullins W W and Sekerka R F 1964 J. Appl. Phys. 35444

Murr L E 1974 Science 183206

Muthukumar M, Ober C K and Thomas E L 1997 Competing interactions and levels of ordering in self-organizing polymeric materials Science 2771225

Naik R R and Stone M O 2005 Mater. Today 18

Naka K and Chujo Y 2001 Chem. Mater. 133245

Nassif N, Pinna N, Gehrke N, Antonetti M, Jager C and Cölfen H 2005 Proc. Natl Acad. Sci. 10212653

Neilson G W, Howe R A and Enderby J E 1975 Chem. Phys. Lett. 33284

Ng K-O and Vanderbilt D 1995 Phys. Rev. B 522177

Noyes R M and Field R J 1974 Annu. Rev. Phys. Chem. 2595

Oettel M, Dominguez A and Dietrich S 2005 J. Phys.: Condens. Matter 17 L337

Orme C A et al 2001 Nature $\mathbf{4 1 1} 775$

Ortoleva P 1980 Symmetries in Science ed B Gruber and R S Millman (New York: Plenum) p 279

Oswald P, Bechhoefer J and Melo F 1991 MRS Bull. 38

Ouyang Q and Swinney H L 1991 Nature 352610

Ozin G A and Arsenault A C 2005 Nanochemistry: A Chemical Approach to Nanomaterials (London: Royal Society of Chemistry Publishing)

Page A J and Sear R P 2006 Phys. Rev. Lett. 97065701

Park R J and Meldrum F C 2004 J. Mater. Chem. 142291

Parker A R et al 2000 J. Opt. A: Pure Appl. Opt. 2 R15

Parsegian V A and Brenner S L 1976 Nature 259632

Parsons D 1968 Funny Ha Ha and Funny Peculiar (London: Pan Books) p 39, quoting The Guardian (date not given). Pawin G, Wong K L, Kwon K-Y and Bartels L 2006 Science 313961

Perez-Munuzuri V et al 1991 Nature 353740

Phillips C G and Kaye S R 1997 Respir. Physiol. 10785

Plass R, Last J A, Bartelt N C and Kellogg G L 2001 Nature 412875

Pohl K, Bartelt M C, de la Figuera J, Bartelt N C, Hrbek J and Hwang R Q 1999 Nature 397238

Popescu D C, van Leeuwen E N M, Rossi N A A, Holder S J, Jansen J A and Sommerdijk N A J M 2006 Angew. Chem. Int. Edn 451762

Price G D 1983 Phys. Chem. Mineral. 1077

Price G D, Parker S C and Yeomans J 1985 Acta Crystallogr. B 41231

Prigogine I 1980 From Being to Becoming: Time and Complexity in the Physical Sciences (San Fransisco: Freeman) Prigogine I 2002 Phil. Trans. A 360299

Puech P-H, Askovic V, de Gennes P-G and Brochard-Wyart F 2006 Biophys. Rev. Lett. 185

Quéré D 2005 Nature 4351168

Radman M 2001 Nature 413115

Rickman J M and Srolovitz D J 1993 Surf. Sci. 284211

Rizzieri R, Mahadevan L, Vazeri A and Donald A 2006 Langmuir 22 3622-6

Ozturk B 2003 Nat. Mater. 2413

Rosei F 2004 J. Phys.: Condens. Matter 16 S1373-436

Royer L 1928 Bull. Soc. Fr. Minéral. Crystallogr. 517

Rubner M 2003 Nature 423925

Sanders D A et al 2004 Phys. Rev. Lett. 93208002

Sass S L and Eyre B L 1973 Phil. Mag. 271447

Saville D A, Chun J, Li J-L, Schniepp H C, Car R and Aksay I A 2006 Phys. Rev. Lett. 96018301

Schultze R D and Asunmaa S K 1970 Prog. Surf. Memb. Sci. 3291

Scott S K 1995 Faraday Discuss. 100 C47

Seul M and Andelman D 1995 Science 267476

Shevchenka E V, Talapin D V, Kotov N A, O’Brien S and Murray C B 2006 Nature 43955

Shirikoff J and Erb U 1994 Phil. Mag. A 701055

Smith J M 1968 Mathematical Ideas in Biology (Cambridge: Cambridge University Press) pp 123-5

Smith J M 1998 Shaping Life: Genes Embryos and Evolution (London: Weidenfeld and Nicolson)

Soai K, Shibata T, Morioka H and Choji K 1995 Nature 378767

Sokolov V I 1986

Stern K H 1954 Chem. Rev. 54 79-99 
Stoneham A M 1971 J. Physique Fl 778

Stoneham A M 1974 UKAEA Report AERE-R7934 ed R S Nelson p 319

Stoneham A M 1975 Fundamental Aspects of Radiation Damage Meeting (Gatlinburg, 1975) U.S. ERD-CONF$751006 \mathrm{P} 1 \mathrm{ed}$ M T Robinson and F W Young (text of an invited paper) p 1121-35

Stoneham A M 1977 Solid State Commun. 24 425-8

Stoneham A M 2003 Mater. Sci. Eng. C 23 235-41

Stoneham A M and Durham P J 1973 J. Phys. Chem. Solids 342127

Stoneham A M and Gavartin J 2007 Mater Sci. Eng. C at press

Stoneham A M and Harding J H 2003 Nat. Mater. 277

Stoneham A M and Jain S C 1995 GeSi Strained Layers and their Applications (Bristol: Institute of Physics Publishing)

Stoneham A M and Torres V J B 1995 unpublished work

Stradner A, Sedgwick H, Cardinaux F, Poon W C K, Egelhauf S U and Schurtenburger P 2004 Nature 432492

Strogatz S 2003 Sync: The Emerging Science of Spontaneous Order (New York: Hyperion)

Subramanian A B, Abkarian M, Mahadevan L and Stone H A 2005 Nature 438930

Summers D W and Whittington S G 1988 J. Phys. A: Math. Gen. 211689

Tang Z, Kotov N A, Magonov S, Shevchenka E V, Talapin D V, Kotov N A, O’Brien S and Murray C B 2006 Nature 43955

Tewary V K 1973a J. Phys. F.: Met. Phys. 31275

Tewary V K 1973b Adv. Phys. 22757

Tewary V K and Bullough R 1972 J. Phys. F.: Met. Phys. 2 L69

Thayer G E, Bartelt N C, Ozolins V, Schmid A K, Chiang S and Hwang R Q 2002 Phys. Rev. Lett. 89036101

Thom R 1983 Mathematical Models of Biogenesis (New York: Wiley)

Thompson D'A 1917 On Growth and Form (Cambridge: Cambridge University Press) (abridged editions 1961 1991)

Thürmer K, Carter C B, Bartelt N C and Hwang R Q 2004 Phys. Rev. Lett. 92106101

Tiller W A 1991 The Science of Crystallization (Cambridge: Cambridge University Press)

Tilley R J D 1977 Nature 269229

Traube H and Essmann U 1966 Phys. Status Solidi 18813

Traube H and Essmann U 1968 Phys. Status Solidi 25373

Travaille A M, Donners J J J M, Gerritsen J W, Sommerdijk N A J M, Nolte R J M and van Kempen H 2002 Adv. Mater. 14492

Travaille A M, Kaptijn L, Verwer P, Huisken B, Elemans J A A W, Nolte J M and van Kempen H 2003 J. Am. Chem. Soc. 12511571

Turin L 1996 Chem. Senses 21773

Turing A M 1952 Phil. Trans. R. Soc. B 23737

Twarock R 2006 Phil. Trans. R. Soc. A 3643357

Ueshima R and Asami T 2003 Nature 425679

Ullmaier H, Zeller R and Dederichs P R 1973 Phys. Lett. A 44331

Vainstein B K 1986 Symmetry: Unifying Human Understanding (Oxford: Pergamon) (also issued as Comput. Math. Appl. B 12237

van der Merwe J H 1991 Sir Charles Frank: An Eightieth Birthday Tribute ed R G Chambers et al (Bristol: Hilger) p 119

van Gastel R, Plass R, Bartelt N C and Kellogg G L 2003 Phys. Rev. Lett. 91055503

van Megen W and Snook I 1976 Nature 262571

van Ommen A H, Koek B H and Viegers M P A 1986 Appl. Phys. Lett. 491062

van Roijen V A et al 1975 Acta Metall. 23987

Viedma C 2005 Phys. Rev. Lett. 94065504

Wagner C 1954 J. Electrochem. Soc. 101225

Wagner C 1956 J. Electrochem. Soc. 103571

Wales D 2003 Energy Landscapes (Cambridge: Cambridge University Press)

Wallace D, Stoneham A M, Testa A, Harker A H and Ramos M M D 1993 Mol. Simul. 2385

Wei C M and Chou M Y 2002 Phys. Rev. B 66233408

Weibel E R 2000 Symmomorphis, on Form and Function in Shaping Life (Harvard: UP)

Weightman P, Dolan G J, Smith C I, Cuquerella M C, Almond N J, Farrell T, Fernig D G, Edwards C and Martin D S 2006 Phys. Rev. Lett. 96086102

Weiner S and Addadi L 1997 J. Mater. Chem. 7689

Wells A F 1986 Phil. Trans. R. Soc. A 319291

Wentzel R N 1936 Ind. Eng. Chem. 28988 
Weyl H 1952 Symmetry (Princeton, NJ: Princeton University Press)

Whitesides G M and Grzybowski B 2002 Science 2952418

Williams P, Keshavarz-Moore E and Dunnill P 1996 Enzyme Microbiol. Technol. 19208

Wilson J A, DiSalvo F J and Mahajan S 1975 Adv. Phys. 24117

van der Merwe J H 1991 Sir Charles Frank: An Eightieth Birthday Tribute (Bristol: Hilger) p 119

Winfree A T 1974 Sci. Am. 23082

Winfree A T 1980 The Geometry of Biological Time (Berlin: Springer)

Winfree A T 1991 Nature 352568

Witten T A and Sander L M 1981 Phys. Rev. Lett. 471400

Woo C H and Frank W 1985 J. Nucl. Mater. 1377

Worthington A M 1908 A Study of Splashes (London: Longmans Green)

Xu L, Zhang W W and Nagel S R 2005 Phys. Rev. Lett. 94184505

Zandi R et al 2004 Proc. Natl Acad. Sci. 10115556

Zeppenfeld P 2004 Surf. Sci. 54952

Zeppenfeld P, Krzyowski M, Romainczyk M, Comsa C and Lagally M G 1994 Phys. Rev. Lett. 722737

Zik et al 1998 Phys. Rev. Lett. 813868 